\title{
Convenient Partial Poisson manifolds
}

\author{
Fernand PELLETIER ${ }^{1}$ and Patrick CABAU ${ }^{2}$
}

\section{Contents}

1 Introduction $\quad 2$

2 Convenient Partial Poisson manifold 3

2.1 Convenient framework . . . . . . . . . . . . . . . . . 3

2.2 Partial Poisson manifold . . . . . . . . . . . . . . . . 4

2.3 Partial Lie algebroid and partial Poisson manifold . . . . . . . . 9

2.4 Almost symplectic foliation associated to a partial Poisson struc-

ture ........................... 14

2.4.1 Preliminaries and notations . . . . . . . . . . . 14

2.4.2 Almost symplectic foliation of a partial Poisson structure 15

2.4.3 Existence of almost symplectic foliation for Poisson Banach manifolds . . . . . . . . . . . 17

3 Direct and inverse limit of partial Poisson Banach manifolds 19

3.1 Direct and inverse limits of linear bundles . . . . . . . . . . 19

3.2 Convenient Poisson morphisms . . . . . . . . . . . . . 20

3.3 Partial Poisson structures on direct and inverse limits . . . . . . 26

3.4 Proof of Theorem 3.3.4 in the case of direct limit . . . . . . 2 28

3.5 Proof of Theorem 3.3.4 in the case of Inverse limit . . . . . . . 31

4 Existence of almost symplectic foliation for direct limit partial Poisson Banach manifolds

\begin{abstract}
We introduce the concept of partial Poisson structure on a manifold $M$ modelled on a convenient space. This is done by specifying a (weak) subbundle $T^{\prime} M$ of $T^{*} M$ and an antisymmetric morphism $P: T^{\prime} M \rightarrow T M$ such that the bracket $\{f, g\}_{P}=-<d f, P(d g)>$ defines a Poisson bracket on a sub-algebra $\mathcal{A}$ of the algebra of smooth functions $f$ on $M$ whose differential $d f$ induces a section of $T^{\prime} M$. In particular, to each such function $f \in \mathcal{A}$ is associated a Hamiltonian vector field $P(d f)$. This notion takes naturally place in the framework of infinite dimensional weak symplectic manifolds and Lie algebroids. After having defined this concept, we will illustrate it by a lot of natural examples. We will also consider the particular situations of direct (resp. inverse) limits of such Banach structures.
\end{abstract}


Finally, we will also give some results on the existence of (weak) symplectic foliations naturally associated to some particular partial Poisson structures.

MSC 2010.- Primary 58A30, 18A30, 46T05; secondary 17B66, 37K30, $22 \mathrm{E} 65$.

Keywords.- Poisson partial manifold; convenient structure; integrable distribution; direct limit; inverse limit; almost Lie Banach algebroid; almost Lie bracket; Koszul connection; anchor range.

${ }^{1}$ Univ. Savoie Mont Blanc, CNRS, LAMA, 73000 Chambéry, France

fernand.pelletier@univ-smb.fr

2 Univ. Savoie Mont Blanc, CNRS, LAMA, 73000 Chambéry, France

patrickcabau@yahoo.fr

\section{Introduction}

The concept of Poisson structure is a fundamental mathematical tool in Mathematical Physics and classical Mechanics (specially in finite dimensions) and, in an infinite dimensional context, in Hydrodynamics, Quantum Mechanics, as a tool for integrating some evolutionary PDEs (for example $\mathrm{KdV}$ ), .... In any of these situations, we have an algebra $\mathcal{A}$ of smooth functions on some manifold $M$ (eventually infinite dimensional) which is provided with a Poisson bracket, i.e. a Lie bracket $\{$,$\} which satisfies the Leibniz property. Moreover, to the$ derivation $g \mapsto\{f, g\}$ in $\mathcal{A}$, we can associate a vector field $X_{f}$ on $M$ called the Hamiltonian vector field of $f$. In infinite dimension, when $M$ is a Banach manifold and $\mathcal{A}=\mathcal{C}^{\infty}(M)$, such a framework was firstly defined and studied in a series of papers by A. Odzijewicz, T. Ratiu and their collaborators (see for instance OdzRat]); we will see how this context is included in our presentation. A more recent approach was also proposed by K.H. Neeb, H. Sahlmann and T. Thiemann ([NeSaTh $]$ ) when $M$ is a smooth manifold modelled on a locally convex topological vector space: the authors consider a subalgebra $\mathcal{A}$ of $C^{\infty}(M)$ which is provided with a Poisson bracket and such that the following separation assumption is satisfied:

$$
\left\{\forall x \in M, \forall f \in \mathcal{A}, d_{x} f(v)=0\right\} \Longrightarrow\{v=0\}
$$

This condition implies that the Hamiltonian field $X_{f}$ is defined for any $f \in \mathcal{A}$.

Our purpose is to propose, in an infinite dimensional context, a Poisson framework for which the Poisson bracket can be defined for some particular local or global smooth functions on $M$.

Essentially we consider:

- a subalgebra $\mathcal{A}(M)$ of the algebra smooth functions $f$ on $M$ whose differential $d f$ induces a section of a subbundle of $T^{\prime} M$ of $T^{*} M$; 
- a bundle morphism $P: T^{\prime} M \rightarrow T M$ such that $\{f, g\}_{P}=d g(P(d f))$ defines a Poisson bracket on $\mathcal{A}$.

After having given a lot of examples of partial Poisson manifolds, we show that the subbundle $T^{\prime} M \rightarrow M$ associated to a partial Poisson manifold can be endowed with a partial Lie algebroid structure and not necessarly with a classical Lie algebroid structure as it can be done in finite dimension.

Finally, in the last section, we look for the existence of a weak symplectic foliation associated to a partial Poisson structure.

\section{Convenient Partial Poisson manifold}

\subsection{Convenient framework}

The convenient setting discovered by A. Frölicher and A. Kriegl (cf. FroKri) gives an adapted framework for differentiation in the spaces we consider here. It coincides with the classical Gâteaux approach on Fréchet spaces.

The references for this section is the tome KriMic which includes some further results and the paper EgeWur.

For short, a convenient vector space $E$ is a locally convex topological vector space (l.c.t.v.s) such that a curve $c: \mathbb{R} \longrightarrow E$ is smooth if and only if $\lambda \circ c$ is smooth for all continuous linear functionals $\lambda$ on $E$. We then get a second topology on $E$ which is the final topology relatively to the set of all smooth curves and called the $c^{\infty}$-topology. This last topology may be different from the l.c.t.v.s topology and, for this topology, $E$ cannot be a topological vector space. However for Fréchet (and so Banach) spaces, both topologies coincide. A map $f: E \rightarrow \mathbb{R}$ is smooth if and only if $f \circ c: \mathbb{R} \rightarrow \mathbb{R}$ is a smooth map for any smooth curve $c$ in $E$.

The convenient calculus provides an appropriate extension of differential calculus to such spaces because, for any $c^{\infty}$-open set $U$ of a convenient space $E$ and any convenient space $F$, we have the following properties:

- the space $C^{\infty}(U, F)$ of smooth maps may be endowed with a structure of convenient space;

- the differential operator $d: C^{\infty}(U, F) \longrightarrow C^{\infty}(U, L(E, F))$ defined by

$$
d f(x) \cdot v=\lim _{t \longrightarrow 0} \frac{f(x+t v)-f(x)}{t}
$$

where $L(E, F)$ denotes the space of all bounded (equivalently smooth) linear mappings from $E$ to $F$, exists and is linear and smooth;

- the chain rule holds.

Therefore the notion of smooth convenient manifold $M$ ([KriMic, 27) modelled on a convenient vector space $\mathbb{M}$ is defined in an obvious way.

The notion of weak submanifold is adapted from [Pel] as follows. 
Definition 2.1.1. A weak submanifold of $M$ is a pair $(N, \varphi)$ where $N$ is a non necessarily Haussdorf convenient connected manifold (modelled on a convenient space $F$ ) and $\varphi: N \longrightarrow M$ is a conveniently smooth map such that:

- there exists a continuous injective linear map $i: F \longrightarrow E$ (for the structure of l.c.v.s. of E)

- $\varphi$ is an injective conveniently smooth map and the tangent map $T_{x} \varphi$ : $T_{x} N \longrightarrow T_{\varphi(x)} M$ is an injective continuous linear map with closed range for all $x \in N$.

The notions of convenient vector bundle ([KriMic, 29) and Lie group ( KriMic], 36) are defined naturally.

\subsection{Partial Poisson manifold}

Let $M$ be a convenient manifold modelled on a convenient space $\mathbb{M}$. We denote by : $p_{M}: T M \rightarrow M$ its kinematic tangent bundle (KriMic, 28.12) and by $p_{M}^{*}: T^{*} M \rightarrow M$ its kinematic cotangent bundle (KriMic, 33.1).

Definition 2.2.1. A vector subbundle $p^{\prime}: T^{\prime} M \rightarrow M$ of $p_{M}^{*}: T^{*} M \rightarrow M$ where $p^{\prime}: T^{\prime} M \rightarrow M$ is a convenient bundle is a weak subbundle of $p_{M}^{*}: T^{*} M \rightarrow M$ if the canonical injection $\iota: T^{\prime} M \rightarrow T^{\prime} M$ is a convenient bundle morphism.

Following [KriMic, 48, for any open set $U$ in $M$ we introduce:

Definition 2.2.2. Let $\mathcal{A}(U)$ be the set of smooth functions $f \in C^{\infty}(U)$ such that each iterated derivative $d^{k} f(x) \in L_{\mathrm{sym}}^{k}\left(T_{x} M, \mathbb{R}\right)\left(k \in \mathbb{N}^{*}\right)$ satisfies:

$$
\forall x \in U, \forall\left(u_{2}, \ldots, u_{k}\right) \in\left(T_{x} M\right)^{k-1}, d_{x}^{k} f\left(., u_{2}, \ldots, u_{k}\right) \in T_{x}^{\prime} M
$$

\section{Remark 2.2.3.}

1. If $T^{\prime} M=T^{*} M$ then for any open set $U$ in $M$ the algebra $C^{\infty}(U)$ satisfies the assumption of Definition 2.2.2 and so in this case $\mathcal{A}(U)=C^{\infty}(U)$.

2. Consider $f \in \mathcal{A}(U)$, then for any $x \in U$, any $k \in \mathbb{N}$ any $u_{2}, \ldots, u_{k}$ in $T_{x} M$, if $A$ is an endomorphism of $T_{x} M$ then the linear map $u \mapsto$ $d_{x}^{k} f\left(A(u), u_{2}, \ldots, u_{k}\right)$ belongs to $T_{x}^{\prime} M$

Proposition 2.2.4. Fix any open set $U$ in $M$,

1. The set $\mathcal{A}(U)$ is a subalgebra of $C^{\infty}(U)$.

2. For each $k \in \mathbb{N}$ and local vector fields $X_{1}, \ldots, X_{k}$ on $U$ the map

$$
x \mapsto d^{k} f\left(X_{1}, \ldots, X_{k}\right)(x)
$$

belongs to $\mathcal{A}(U)$. 
Proof. It is clear that $\mathcal{A}(U)$ is a real vector subspace of $C^{\infty}(U)$.

We must show that for any $f, g \in \mathcal{A}(U)$ then the product $f g$ belongs to $\mathcal{A}(U)$. We fix such $f, g \in \mathcal{A}(U)$. Since $d f$ and $d g$ are sections of $T^{\prime} M$ on $U$, it follows $d_{x}(f g)$ belongs to $T_{x}^{\prime} M$ for all $x \in U$. More generally for any $k \in \mathbb{N}$, any $x \in U$ and any $u_{2}, \ldots, u_{k}$ in $T_{x} M$ we have the following formulae for the derivative

$$
\begin{aligned}
& d_{x}^{k}(f g)\left(., u_{2}, \ldots, u_{k}\right) \\
= & \sum_{\substack{l=1 \\
k}}\left(\sum_{\sigma} d_{x}^{l} f\left(u_{\sigma_{1}}, \ldots, u_{\sigma_{l}}\right) d_{x}^{k-l} g\left(., u_{\sigma_{l+1}}, \ldots, u_{\sigma_{k-1}}\right)\right. \\
& \left.+d_{x}^{l} g\left(u_{\sigma_{1}}, \ldots, u_{\sigma_{l}}\right) d f_{x}^{k-l}\left(., u_{\sigma_{l+1}}, \ldots, u_{\sigma_{k-1}}\right)\right)
\end{aligned}
$$

where the summation on $\sigma$ is for all $(l, k-l-1)$ shuffles $\sigma$ of $\{1, \ldots, l, \ldots, k-1\}$ Since $f$ and $g$ belongs to $\mathcal{A}(U)$, it follows that the second member of the previous relation is a section of $T_{x}^{\prime} M$ which ends the proof of the first part.

Fix some vector fields $X_{1}, \ldots X_{k}$ on $U$ and we set $\phi=d^{k} f\left(X_{1}, \ldots, X_{k}\right)$.

The differential $d_{x}^{l} \phi\left(u_{1}, \ldots, u_{l}\right)$ is a sum of terms of type

$$
d_{x}^{k+h_{0}} f\left(u^{\sigma^{0}}, X_{1}^{h_{1}}\left(u^{\sigma^{1}}\right), \ldots X_{j}^{h_{j}}\left(u^{\sigma^{j}}\right), \ldots X^{h_{k}}\left(u^{\sigma^{k}}\right)\right)
$$

with the following notations and constraints:

- $h_{0}+h_{1}+\cdots+h_{k}=k+h$ where $h \in\{0, \ldots, l-k\}$;

- for $j \in\{0, \ldots, k\}$,

- if $h_{j}=0$ then $\sigma^{j}$ is an empty set,

- if $h_{j}>0$ then $\sigma^{j}$ is a strictly increasing sequence $\sigma^{j}=\left(\sigma_{1}^{j}, \ldots, \sigma_{h_{j}}^{j}\right)$ and $u^{\sigma^{j}}=\left(u_{\sigma_{1}^{j}}, \ldots u_{\sigma_{h_{j}}^{j}}\right)$;

- for $j \in\{1, \ldots, k\}$,

- if $h_{j}=0$ then $X_{j}^{0}\left(u^{\sigma^{j}}\right)=X_{j}(x)$ (i.e. $\sigma^{j}$ is an empty set),

- if $h_{j}>0$ then $X_{j}^{h_{j}}\left(u^{\sigma^{j}}\right)=T_{x}^{h_{j}} X_{j}\left(u_{\sigma_{1}^{j}}, \ldots, u_{\sigma_{h_{j}}^{j}}\right)$;

- $\left\{\left\{\sigma_{1}^{j}, \ldots \sigma_{h_{j}}^{j}\right\}, j \in\{0, \ldots k\}, \sigma^{j} \neq \emptyset\right\}$ is a partition of $\{1, \ldots, l\}$.

Note that, according to the previous conditions, a term of type (2.2) is uniquely defined by the partition

$$
\left\{\left\{\sigma_{1}^{j}, \ldots \sigma_{h_{j}}^{j}\right\}, j=0, \ldots k, \sigma^{j} \neq \emptyset\right\}
$$

and $d_{x}^{l} \phi\left(u_{1}, \ldots, u_{l}\right)$ is the sum of terms of type (2.2) for all such partitions. 
Now, if we delete $u_{1}$, in $d_{x}^{l} \phi\left(u_{1}, \ldots, u_{l}\right)$, then, each term of type (2.2) will give rise to a term in one and only one of the following situations (according to the associated partition):

(i) there exists $1 \leq i \leq h_{0}$ with $\sigma_{i}^{0}=1$. Then in all terms associated to such a partition, after having deleted $u_{1}$, we obtain a term of type

$$
d_{x}^{k+h_{0}} f\left(u_{\sigma_{1}^{0}}, \ldots, \widehat{u_{\sigma_{i}^{0}}}, \ldots, u_{\sigma_{h_{0}}^{0}}, X_{1}^{h_{1}}\left(u^{\sigma^{1}}\right), \ldots X_{j}^{h_{j}}\left(u^{\sigma^{j}}\right), \ldots X_{k}^{h_{k}}\left(u^{\sigma^{k}}\right)\right)
$$

which belongs to $T_{x}^{\prime} M$, according to the characterization of $\mathcal{A}(U)$ and the symmetry of $d_{x} f^{k+l}$;

(ii) there exists $1 \leq j \leq k$ such that some $\sigma_{i}^{j}=1$. Therefore, after deleting $u_{1}$ in $X_{j}^{\sigma^{j}}\left(u^{\sigma^{j}}\right)$, we obtain $T_{x}^{h_{j}} X_{j}\left(u_{\sigma_{1}^{j}}, \ldots, \widehat{u_{\sigma_{i}^{j}}}, \ldots, u_{\sigma_{h_{j}}^{j}}\right)$ which defines an endomorphism of $T_{x} M$ and all the other terms $X_{i}^{h_{i}}\left(u^{\sigma^{i}}\right)$, for $i \neq j$, belong to $T_{x} M$. Again, by same arguments in (i) and Remark 2.2.3, 2., in all terms associated to such a partition, the value of

$$
d_{x}^{k+h_{0}} f\left(u^{\sigma^{0}}, X_{1}^{h_{1}}\left(u^{\sigma^{1}}\right), \ldots X_{j}^{h_{j}}\left(u^{\sigma^{j}}\right), \ldots X^{h_{k}}\left(u^{\sigma^{k}}\right)\right)
$$

after deleting $u_{\sigma_{i}^{j}}$, belongs to $T_{x}^{\prime} M$.

This implies that $d_{x} \phi\left(., u_{2}, \ldots, u_{l}\right)$ belongs to $T_{x}^{\prime} M$ for any $u_{2}, \ldots, u_{l}$ in $T_{x} M$.

Since $d_{x}^{l} \phi\left(u_{1}, u_{2}, \ldots, u_{l}\right)$ is symmetric in $\left(u_{1}, \ldots, u_{l}\right)$, it follows that $d_{x}^{l} \phi\left(u_{1}, \ldots, \widehat{u_{i}}, \ldots, u_{l}\right)$ also belongs to $T_{x}^{\prime} M$ for any $i \in\{1, \ldots, l\}$. Now, as $d_{x}^{k+l} f$ and $T_{x}^{h_{j}} X_{j}$ for $j \in\{1, \ldots, k\}$, are bounded symmetric maps, it follows that $d_{x}^{l} \phi$ is also a bounded symmetric map.

Finally, such a result is true for any integer $k$ and $l$, any $x \in U$ and any local vector fields $X_{1}, \ldots, X_{k}$ on $U$ and so the proof is complete.

Consider the canonical bilinear crossing $<,>$ between $T^{*} M$ and $T M$.

Definition 2.2.5. A morphism $P: T^{\prime} M \rightarrow T M$ is called skew-symmetric if it satisfies the relation

$$
<\xi, P(\eta)>=-<\eta, P(\xi)>
$$

for $\xi$ and $\eta$ of $T_{x}^{\prime} M$. We say that $P$ is an almost Poisson anchor.

Given such a morphism $P$, on $\mathcal{A}(U)$ we define:

$$
\{f, g\}_{P}=-<d f, P(d g)>
$$

In these conditions, the relation (2.4) defines a skew-symmetric bilinear map $\{., .\}_{P}: \mathcal{A}(U) \times \mathcal{A}(U) \rightarrow C^{\infty}(U)$. 
Lemma 2.2.6. The bilinear map $\{., .\}_{P}$ takes values in $\mathcal{A}(U)$ and satisfies the Leibniz property:

$$
\{f, g h\}_{P}=g\{f, h\}_{P}+h\{f, g\}_{P}
$$

Proof. Since $\{f, g\}_{P}=-d f(P(d g))$, for all $f$ and $g$ in $\mathcal{A}(U)$, from Proposition 2.2.4 2., it follows that $d f(P(d g))$ belongs to $\mathcal{A}(U)$.

Finally, from the definition of the bracket, we have

$$
\{f, g h\}_{P}=-<d f, P d(g h)>=-g<d f, p(d h)>-h<d f, P(d g)>=g\{f, h\}_{P}+h\{f, g\}_{P}
$$

and so the proof is complete.

Definition 2.2.7. Let $p^{\prime}: T^{\prime} M \rightarrow M$ be a weak subbundle of $p_{M}^{*}: T^{*} M \rightarrow M$ and $P: T^{\prime} M \rightarrow T M$ an almost Poisson anchor.

1. We say that $\left(T^{\prime} M, M, P,\{., .\}_{P}\right)$ is a partial Poisson structure on $M$ if the bracket $\{., .\}_{P}$ satisfies the Jacobi identity

$$
\left\{f,\{g, h\}_{P}\right\}_{P}+\left\{g,\{h, f\}_{P}\right\}_{P}+\left\{h,\{f, g\}_{P}\right\}_{P}=0 .
$$

In this case $P$ is called a Poisson anchor

2. Let $\mathcal{A}$ be a subalgebra of $\mathcal{A}(M)$ such that the restriction of $\{., .\}_{P}$ to $\mathcal{A} \times \mathcal{A}$ takes values in $\mathcal{A}$. We say that $\mathfrak{A}$ is a Poisson subalgebra of $\mathfrak{A}(M)$ and $\left(M, \mathfrak{A},\{., .\}_{P}\right)$ is a partial Poisson manifold

Note that in particular, if $\left(T^{\prime} M, M, P,\{., .\}_{P}\right)$ is a partial Poisson structure, then $\left(M, \mathcal{A}(M),\{., .\}_{P}\right)$ is always a partial Poisson manifold.

Definition 2.2.8. Let $\left(M_{1}, \mathcal{A}_{1},\{., .\}_{P_{1}}\right)$ and $\left(M_{2}, \mathcal{A}_{2},\{., .\}_{P_{2}}\right)$ two partial Poisson manifolds. A smooth map $\phi: M_{1} \rightarrow M_{2}$ is called a Poisson map if the induced map $\phi^{*}: \mathcal{C}^{\infty}\left(M_{2}\right) \rightarrow \mathcal{C}^{\infty}\left(M_{1}\right)$, defined by $\phi^{*}(f):=f \circ \phi$, is such that

$$
\left\{\begin{aligned}
\varphi^{*}\left(\mathcal{A}_{2}\right) & \subset \mathcal{A}_{1} \\
\left\{\varphi^{*}(f), \varphi^{*}(g)\right\}_{P_{1}} & =\varphi^{*}\left(\{f, g\}_{P_{2}}\right)
\end{aligned}\right.
$$

If $M$ is a Hilbert (resp. Banach, resp. Fréchet) manifold and if the weak subbundle $T^{\prime} M$ is a Hilbert (resp. Banach, resp. Fréchet) bundle, the partial Poisson manifold $\left(M, \mathcal{A},\{., .\}_{P}\right)$ will be called a partial Poisson Hilbert (resp. Banach, resp Fréchet) manifold.

As classically, given a partial Poisson manifold $\left(M, \mathcal{A},\{., .\}_{P}\right)$, any function $f \in \mathcal{A}$ is called a Hamiltonian and the associated vector field $X_{f}=P(d f)$ is called a Hamiltonian vector field.

We then have $\{f, g\}=X_{f}(g)$ and also $\left[X_{f}, X_{g}\right]=X_{\{f, g\}}$ (see NeSaTh), which is equivalent to

$$
P(d\{f, g\})=[P(d f), P(d g)]
$$


Example 2.2.9. Finite dimensional Poisson manifold. - A finite dimensional Poisson manifold $\left(M, \mathcal{C}^{\infty}(M),\{.,\}.\right)$ (cf. [Marl]) is a particular case of partial Poisson manifold. Indeed, to each function $f$ on $M$ is associated a Hamiltonian vector field $X_{f}$. Since $T^{\prime} M=T^{*} M$ is locally generated by differential of functions, therefore the map $d f \mapsto X_{f}$ extends to a unique skew-symmetric morphism of bundles $P: T^{*} M \rightarrow T M$ such that $P(d f)=X_{f}$.

Example 2.2.10. Banach-Poisson manifold. - Let $M$ be a Banach manifold. The notion of Banach-Poisson manifold was defined and developed in [OdzRat] and [Rat]. These authors assume that there exists a Poisson bracket $\{.,$.$\} on$ $C^{\infty}(M)$ such that to each linear functional $\xi$ on $M$ is associated a section $\xi^{\prime}$ of the bidual $T^{\prime \prime} M$ which, in fact, belongs to $T M \subset T^{* *} M$. This gives rise to a skew-symmetric morphism $P: T^{\prime} M \rightarrow T M$. Conversely, given such a morphism, we get a bracket $\{.,$.$\} on C^{\infty}(M)$ as given in (2.4). Therefore, if this bracket satisfies the Jacobi identity, we get the previous notion of BanachLie Poisson manifold (see [Pel]]). Therefore $M$ is endowed with a partial Poisson structure.

Example 2.2.11. Banach-Poisson-Lie algebroid.-A Banach-Lie algebroid is a Banach bundle $\pi: E \rightarrow M$ provided with a morphism $\rho: E \rightarrow T M$ (anchor) and a Lie bracket $[., .]_{E}$ which is a skew-symmetric bilinear map $\Gamma(E) \times \Gamma(E) \rightarrow$ $\Gamma(E)$ such that

$$
[X, f Y]_{E}=d f(\rho(X)) Y+f[X, Y]_{E}
$$

and $\Gamma(E)$ is the set of sections of $\pi: E \rightarrow M$ which satisfy the Jacobi identity (see [Ana] and [CabPel1]).

If $\pi^{*}: E^{*} \rightarrow M$ denotes the dual bundle of $\pi: E \rightarrow M$, there exists a Banach subbundle $T^{\prime} E^{*}$ of $T^{*} E^{*}$ defined as follows.

For any (local) section $s: U \rightarrow E_{\mid U}$ we denote by $\Phi_{s}$ the linear map on $E_{\mid U}^{*}$ defined by $\Phi_{x}(\xi)=<\xi, s \circ \pi^{*}(\xi)>$. Then for any $\sigma \in E^{*}$ then $T_{\sigma}^{\prime} E^{*}$ is generated by the set

$\left\{d\left(\Phi_{s}+f \circ \pi *\right), \quad s\right.$ any section of $E_{\mid U}, \quad f \in C^{\infty}(U), \quad U$ any neighbourhood of $\left.\pi^{*}(\sigma)\right\}$.

Let $\mathcal{A}_{L}\left(E^{*}\right)$ be the set of smooth functions $f: E^{*} \rightarrow \mathbb{R}$ whose restriction to each fiber is linear. Clearly $\mathcal{A}_{L}\left(E^{*}\right)$ is contained in $\mathcal{A}\left(E^{*}\right)$.

Now there exists a morphism $P: T^{\prime} E^{*} \rightarrow T E^{*}$ which gives rise to a bracket $\{., .\}_{P}$ whose restriction to $\mathcal{A}_{L}\left(E^{*}\right) \times \mathcal{A}_{L}\left(E^{*}\right)$ takes values in $\mathcal{A}_{L}\left(E^{*}\right) 1$. Since the Lie bracket $[., .]_{E}$ satisfies the Jacobi identity, this implies that the bilinear map $\{., .\}_{P}$ also satisfies the Jacobi identity; in this way, we obtain a Poisson manifold $\left(E^{*}, \mathcal{A}_{L}\left(E^{*}\right),\{., .\}_{P}\right)$ (see [CabPel1] for more details).

Example 2.2.12. Weak symplectic convenient manifold. - A weak symplectic manifold is a convenient manifold $M$ endowed with a closed 2 -form $\omega$ such that the associated morphism

$$
\begin{array}{cl}
\omega^{b}: T M & \rightarrow T^{*} M \\
X & \mapsto \omega(X, .)
\end{array}
$$

\footnotetext{
${ }^{1}$ such a Poisson bracket is called a Linear Poisson bracket
} 
is injective (see [KriMic], 48). Therefore if the range $T^{\prime} M=\omega^{b}(T M)$ is a weak subbundle of $T^{*} M$ we have a skew-symmetric morphism $P=\left(\omega^{b}\right)^{-1}: T^{\prime} M \rightarrow$ TM. According to [KriMic] $\S 48$, we have a bracket which satisfies the Leibniz property on $\mathcal{A}(M)$. Moreover, since $\omega$ is closed, this bracket satisfies the Jacobi identity (see [KriMic], Theorem 48.8); so we obtain a partial Poisson structure on $M$.

Remark 2.2.13. If $M$ is a weak symplectic Banach manifold endowed with a symplectic 2-form $\omega$, then the range $T^{*} M=\omega^{b}(T M)$ is not always a weak subbundle of $T^{*} M$ (cf. Marsden example in [Mars]). However, on $M$ we have a subalgebra $\mathcal{A}(M)$ of $C^{\infty}(M)$ which has the same characterization as in Definition [2.2.2. Then in [KriMic], 48 the authors shows that can be provided with a Lie Poisson bracket. The reader will find a generalization of such a situation in Banach setting in [Tum].

Remark 2.2.14. One can also find in $[\mathrm{Kol}]$ how $C^{\infty}\left(\mathbb{S}^{1}\right)$ can be endowed with a weaker structure than a partial Poisson one: since the product of two functionals is no well defined ( $c f$. [Olv], p. 357), the Leibniz rule has no counterpart in this situation.

\subsection{Partial Lie algebroid and partial Poisson manifold}

We begin this section by adapting the concept of almost Banach Lie algebroid to the convenient setting.

Let $\pi: E \rightarrow M$ be a convenient vector bundle on a convenient manifold modelled on a convenient space $\mathbb{M}$ whose fiber is modelled on a convenient space $\mathbb{E}$.

Definition 2.3.1. A morphism of vector bundles $\rho: E \rightarrow T M$ is called an anchor. The triple $(E, M, \rho)$ is called an anchored bundle.

Let $\Gamma(E)$ be the $\mathcal{C}^{\infty}(M)$-module of smooth sections of $E \rightarrow M$. The morphism $\rho$ gives rise to a morphism (again denoted $\rho) \rho: \Gamma(E) \rightarrow \Gamma(T M)=\mathfrak{X}(M)$.

Definition 2.3.2. An almost Lie bracket on an anchored bundle $(E, M, \rho)$ is a bilinear map $[,]_{E}: \Gamma(E) \times \Gamma(E) \longrightarrow \Gamma(E)$ which satisfies the following properties:

1. $[,]_{E}$ is antisymmetric and depends only on 1-jets of sections

2. Leibniz property:

$$
\forall s_{1}, s_{2} \in \Gamma(E), \forall f \in C^{\infty}(M),\left[s_{1}, f s_{2}\right]_{E}=f .\left[s_{1}, s_{2}\right]_{E}+d f\left(\rho\left(s_{1}\right)\right) . s_{2} .
$$

The quadruple $\left(E, M, \rho,[,]_{E}\right)$ is called an almost algebroid. 
Definition 2.3.3. Given an almost Lie bracket $[,]_{E}$, the Jacobiator is the tensororial map $J_{E}: \Gamma(E)^{3} \rightarrow \Gamma(E)$ defined, for all $s_{1}, s_{2}, s_{3} \in \Gamma(E)$ by

$$
J_{E}\left(s_{1}, s_{2}, s_{3}\right)=\left[s_{1},\left[s_{2}, s_{3}\right]_{E}\right]_{E}+\left[s_{2},\left[s_{3}, s_{1}\right]_{E}\right]_{E}+\left[s_{3},\left[s_{1}, s_{2}\right]_{E}\right]_{E}
$$

A convenient Lie algebroid is an almost Lie algebroid $\left(E, M, \rho,[,]_{E}\right)$ such that the associated Jacobiator $J_{E}$ is vanishes identically and $\rho$ is a Lie algebra morphism from $\Gamma(E)$ to $\mathfrak{X}(M)$.

Proposition 2.3.4. Consider a convenient almost Lie algebroid $\left(E, \pi, M, \rho,[., .]_{E}\right)$.

1. For any open set $U \subseteq M$ and any $\left(s_{1}, s_{2}\right) \in \Gamma\left(E_{U}\right)^{2}$, the map

$$
\left(s_{1}, s_{2}\right) \mapsto \rho\left(\left[s_{1}, s_{2}\right]_{E}\right)-\left[\rho\left(s_{1}\right), \rho\left(s_{2}\right)\right]
$$

only depends on the 1-jet of $\rho$ at any $x \in U$ and the values of $s_{1}$ and $s_{2}$ at $x$.

2. If the Jacobiator $J_{E_{U}}$ vanishes identically, then we have:

$$
\forall\left(s_{1}, s_{2}\right) \in \Gamma\left(\mathcal{A}_{U}\right)^{2}, \rho\left(\left[s_{1}, s_{2}\right]_{\mathcal{A}}\right)=\left[\rho\left(s_{1}\right), \rho\left(s_{2}\right)\right] .
$$

3. If property (2.6) is true, then $J_{\mathcal{A}_{U}}$ is a bounded trilinear $C^{\infty}(U)$ morphism from $\Gamma\left(\mathcal{A}_{U}\right)^{3}$ to $\Gamma\left(\mathcal{A}_{U}\right)$ which take values in $\operatorname{ker} \rho$ over $U$.

Proof.

(1) Since Point (1) is local, we may assume that $M$ is a $c^{\infty}$-open set in $\mathbb{M}$ and $E=M \times \mathbb{E}$. In this context, according to the Definition 2.3.2, we have

$$
\left[s_{1}, s_{2}\right]_{\mathcal{A}}=d s_{2}\left(\rho\left(s_{1}\right)\right)-d s_{1}\left(\rho\left(s_{2}\right)\right)+C\left(s_{1}, s_{2}\right) .
$$

On the other hand, the Lie bracket of the vector fields $\rho\left(s_{1}\right)$ and $\rho\left(s_{2}\right)$ is:

$$
\left[\rho\left(s_{1}\right), \rho\left(s_{2}\right)\right]=d \rho\left(\rho\left(s_{1}\right), s_{2}\right)-d \rho\left(\rho\left(s_{2}\right), s_{1}\right)+\rho \circ d s_{2}\left(\rho\left(s_{1}\right)\right)-\rho \circ d s_{1}\left(\rho\left(s_{2}\right)\right) \text {. }
$$

We then obtain:

$$
\rho\left(\left[s_{1}, s_{2}\right]_{E}\right)-\left[\rho\left(s_{1}\right), \rho\left(s_{2}\right)\right]=\rho\left(C\left(s_{1}, s_{2}\right)\right)-d \rho\left(\rho\left(s_{1}\right), s_{2}\right)+d \rho\left(\rho\left(s_{2}\right), s_{1}\right) .
$$

From the last member, It follows that $\rho\left(\left[s_{1}, s_{2}\right]_{E}\right)-\left[\rho\left(s_{1}\right), \rho\left(s_{2}\right)\right]$ depends only on the 1 -jet of $\rho$ at any $x \in U$ and the values of $s_{1}$ and $s_{2}$ at $x$.

(2) For any triple $\left(s_{1}, s_{2}, s_{3}\right) \in\left(\Gamma\left(\mathcal{A}_{U}\right)\right)^{3}$ and any $f \in C^{\infty}(U)$, we have (cf. for instance [DuZu] Lemma 8.1.4):

$$
\begin{aligned}
J_{E_{U}}\left(s_{1}, s_{2}, f s_{3}\right)= & {\left[s_{1},\left[s_{2}, f s_{3}\right]_{E}\right]_{E}+\left[s_{2},\left[f s_{3}, s_{1}\right]_{E}\right]_{E}+\left[f s_{3},\left[s_{1}, s_{2}\right]_{E}\right]_{E} } \\
= & \rho\left(s_{1}\right)\left(\rho\left(s_{2}\right)(f) s_{3}+\rho\left(s_{2}\right)(f)\left[s_{1}, s_{3}\right]_{E}\right. \\
& +\rho\left(s_{1}\right)(f)\left[s_{2}, s_{3}\right]_{E}+f\left[s_{1},\left[s_{2}, s_{3}\right]_{E}\right]_{E} \\
& +\rho\left(s_{2}\right)(f)\left[s_{3}, s_{1}\right]_{E}+f\left[s_{2},\left[s_{3}, s_{1}\right]_{E}\right]_{E} \\
& +f\left[s_{3},\left[s_{1}, s_{2}\right]_{E}\right]_{E}-\rho\left(\left[s_{1}, s_{2}\right]_{E}\right)(f) s_{3} \\
= & \left(\left[\rho\left(s_{1}\right), \rho\left(s_{2}\right)\right]-\rho\left(\left[s_{1}, s_{2}\right]_{E}\right)\right)(f) s_{3}+f J_{E_{U}}\left(s_{1}, s_{2}, s_{3}\right)
\end{aligned}
$$


Assume that $J_{E_{U}}$ vanishes on $\Gamma\left(E_{U}\right)^{3}$.

It follows that

$$
\forall\left(s_{1}, s_{2} s_{3}\right) \in\left(\Gamma\left(E_{\mid U}\right)\right)^{3}, \forall f \in C^{\infty}(U), J_{E_{U}}\left(s_{1}, s_{2}, f s_{3}\right)=f J_{E_{U}}\left(s_{1}, s_{2}, s_{3}\right)
$$

if and only if

$\forall\left(s_{1}, s_{2}\right) \in \Gamma\left(\mathcal{A}_{U}\right)^{2}, \forall s_{3} \in \Gamma\left(E_{U}\right), \forall f \in C^{\infty}(U),\left(\left[\rho\left(s_{1}\right), \rho\left(s_{2}\right)\right]-\rho\left(\left[s_{1}, s_{2}\right]_{E}\right)(f) s_{3}=0\right.$

which is equivalent to

$$
\forall\left(s_{1}, s_{2}\right) \in \Gamma\left(E_{U}\right)^{2}, \forall f \in C^{\infty}(U),\left(\left[\rho\left(s_{1}\right), \rho\left(s_{2}\right)\right]-\rho\left(\left[s_{1}, s_{2}\right]_{E}\right)(f)=0\right.
$$

which is equivalent to

$$
\forall\left(s_{1}, s_{2}\right) \in \Gamma\left(E_{U}\right)^{2},\left(\left[\rho\left(s_{1}\right), \rho\left(s_{2}\right)\right]-\rho\left(\left[s_{1}, s_{2}\right]_{E}\right)=0 .\right.
$$

(3) If the property (2.6) is true, then, from (2.8), we have

$$
\forall\left(s_{1}, s_{2}, s_{3}\right) \in\left(\Gamma\left(E_{\mid U}\right)\right)^{3}, \forall f \in C^{\infty}(U), J_{E_{U}}\left(s_{1}, s_{2}, f s_{3}\right)=f J_{E_{U}}\left(s_{1}, s_{2}, s_{3}\right) .
$$

But, we have $\rho \circ J_{E_{U}} \equiv 0$ according to the Jacobi property of the Lie bracket of vector fields and property (2.6). Finally, on the one hand, from the second member of (2.7) since the differential is a bounded morphism of convenient space, (cf. KriMic, Theorem 3.18) and $x \mapsto C_{x}(.,$.$) is a smooth field of a$ bounded linear operators and so is a bounded convenient operator from $\Gamma\left(E_{U}\right)^{2}$ to $\Gamma\left(E_{U}\right)$ and, on the other hand, from its definition, it follows that $J_{E_{U}}$ is a convenient bounded operator from $\Gamma\left(E_{U}\right)^{3}$ to $\Gamma\left(E_{U}\right)$. This completes the proof according to the uniform boundedness principle in KriMic, Proposition 30.1.

Let $(E, \pi, M, \rho)$ be a convenient anchored bundle. Given a sheaf $\mathcal{E}_{M}$ of of subalgebra of the sheaf $C_{M}^{\infty}$ of smooth functions on $M$, let $\mathfrak{P}_{M}$ be a sheaf of $\mathcal{E}_{M}$ modules of section of $E$. Assume that $\mathfrak{P}_{M}$ can be provided with a structure of Lie algebras sheaf which satisfies, for any open set $U$ in $M$ :

- the Lie bracket $[., .]_{\mathfrak{P}(U)}$ on $\mathfrak{P}(U)$ only depends on the 1-jets of sections of $\mathfrak{P}(U)$

for any $\left(s, s^{\prime}\right) \in(\mathfrak{P}(U))^{2}$ and any $f \in \mathcal{E}(U)$, we have the compatibility conditions

$$
\left[s, f s^{\prime}\right]_{\mathfrak{P}(U)}=d f(\rho(s)) s^{\prime}+f\left[s, s^{\prime}\right]_{\mathfrak{P}(U)} .
$$

- $\rho$ induces a Lie algebra morphism from $\mathfrak{P}(U)$ to $\mathfrak{X}(U)$, for any open set $U$ in $M$. 
Then $\left(E, \pi, M, \rho, \mathfrak{P}_{M}\right)$ is called a convenient partial Lie algebroid. The family $\left\{[., .]_{\mathfrak{P}(U)}, U\right.$ open set in $\left.M\right\}$ is called a sheaf bracket and is denoted $[., .]_{E}$.

A partial convenient Lie algebroid $\left(E, \pi, M, \rho, \mathfrak{P}_{M}\right)$ is called strong if for any $x \in M$, the stalk

$$
\mathfrak{P}_{x}=\lim _{\longrightarrow}\left\{\mathfrak{P}(U), \varrho_{V}^{U}, \quad U \text { open neighbourhood of } x\right\}
$$

is equal to $\pi^{-1}(x)$ for any $x \in M$.

Remark 2.3.5. Let $\left(E, \pi, M, \rho, \mathfrak{P}_{M}\right)$ be a convenient partial Lie algebroid. On each open set $U$, we can define the Jacobiator $J_{\mathfrak{P}(U)}$ on sections of $\mathfrak{P}(U)$. Thus, by same arguments in the proof of Proposition 2.3.4. 3., applied for sections in $\mathfrak{P}(U)$ and functions in $\mathcal{E}(U)$, we have $J_{\mathfrak{P}(U)}\left(s_{1}, s_{2}, f s_{3}\right)=f J_{\mathfrak{P}(U)}\left(s_{1}, s_{2}, f s_{3}\right)$ for any $s_{1}, s_{2}, s_{3} \in \mathfrak{P}(U)$ and function $f \in \mathcal{E}(U)$.

This notion of strong partial Lie algebroid is justified by the following result:

Proposition 2.3.6. Let $\left(T^{\prime} M, P,\{.,\}_{P}\right)$ be a partial Poisson structure and we denote by $\mathfrak{P}_{M}$ the sheaf of $\mathcal{A}(U)$-modules generated by the set $\{d f, f \in \mathcal{A}(U)\}$. Then we have the following properties:

1. We can define a sheaf of almost brackets $[.,]_{P}$ on the sheaf $\mathfrak{P}_{M}$ by:

$$
[\alpha, \beta]_{P}=L_{P(\alpha)} \beta-L_{P(\beta)} \alpha-d<\alpha, P(\beta)>
$$

for any open set $U$ in $M$ and any section $\alpha$ and $\beta$ in $\mathfrak{P}(U)$ where $L_{X}$ is the Lie derivative.

Moreover $[., .]_{P}$ satisfies:

$$
\forall(f, g) \in(\mathcal{A}(U))^{2},[d f, d g]_{P}=d\{f, g\}_{P}
$$

2. $\left(\mathfrak{P}_{M},[., .]_{P}\right)$ is a sheaf of Poisson-Lie algebra. In particular, $\left(T^{\prime} M, p_{M}^{\prime}, M, P, \mathfrak{P}_{M}\right)$ is a strong partial convenient Lie algebroid

Proof.

(1) At first we must show that on an open set $U$, the bracket $[., .]_{P}$ takes values in $\mathfrak{P}(U)$. Now any $\alpha \in \mathfrak{P}(U)$ can be written

$$
\alpha=\sum_{i \in I} g_{i} d f_{i}
$$

where $I$ is a finite set of indexes and where each $f_{i}$ and $g_{i}$ belongs to $\mathcal{A}(U)$.

Recall that, for a smooth functions $f$ and $g$ on $U$, and vector field $Y$, we have

$$
L_{g Y} d f=d f(Y) d g+g d^{2} f(Y, .) .
$$

Thus, from Proposition 2.2.4 if $f$ and $g$ belongs to $\mathcal{A}(U)$, also $d f(Y)$ belongs to $\mathcal{A}(U)$ and and $d^{2} f(Y,$.$) is a section of T^{\prime} M$ so $L_{g Y} d f$ belongs to $\mathfrak{P}(U)$. 
It follows that if $\alpha, \beta$ belongs to $\mathfrak{P}(U)$ then $L_{P(\alpha)} \beta$ belongs to $\mathfrak{P}(U)$.

Now we have:

$$
d<\alpha, P(\beta)>=\sum_{i \in I} d g_{i}(P(\beta)) d f_{i}+g_{i} d<d f_{i}, P(\beta)>
$$

But from Proposition 2.2.4, since $f_{i}$ belongs to $\mathcal{A}(U)$ then $d f_{i}(P(\beta))$ belongs to $\mathcal{A}(U)$ and $d<d f_{i}, P(\beta)>=d^{2} f_{i}\left(P(\beta,\right.$.$) is a section of T^{\prime} M$ over $U$. It follows that $d<\alpha, P(\beta)>$ also belongs to $\mathfrak{P}(U)$. Therefore $[., .]_{P}$ takes values in $\mathfrak{P}(U)$ and is skew-symmetric and depends only on the 1-jets of $\alpha$ and $\beta$ The Leibniz property of $[., .]_{P}$ is a direct consequence of the Leibniz property of $\{., .\}_{P}$ and the decomposition of 1 -forms in $\mathfrak{P}_{M}$.

From the definition of $[., .]_{P}$, we have

$[d f, d g]_{P}=d<d f, P d g>-d<d g, P d f>-d<d f, P d g>=-d<d f, P d g>=d\{f, g\}$

which ends the proof of (1).

(2) From Point 1, the bracket (2.11) takes value in $\mathfrak{P}(U)$.

Now we have

$$
\left[d f,[d g, d h]_{P}\right]_{P}=d\left\{f,\{g, h\}_{P}\right\}_{P}
$$

Since the Poisson bracket satisfies the Jacobi identity, the previous relation implies

$$
J_{P}\left(d f_{1}, d f_{2}, d f_{3}\right)=0
$$

for all $f_{2}, f_{2}, f_{3}$ in $\mathcal{A}(U)$. On the other hand, from (2.11) and (2.5) it follows that

$$
P\left(\left[d f_{1}, d f_{2}\right]_{P}\right)=\left[P\left(d f_{1}\right), P\left(d f_{2}\right)\right]
$$

for any $f_{1}, f_{2}$ in $\mathcal{A}(U)$.

According to Remark 2.3.5, for any functional linear combination $\sum_{i=1}^{n} g_{i} d f_{i}$, where $f_{i}, g_{i}$ belongs to $\mathcal{A}(U)$ for $i=1, \ldots, n$, we have

$$
J_{P}\left(d h, d g \sum_{i=1}^{n} g_{i} d f_{i}\right)=\sum_{i=1}^{n} g_{i} J_{P}\left(d g, d h, d f_{i},\right)=0
$$

for any $g, h \in \mathcal{A}(U)$. From the skew symmetry of $J_{P}$.

this implies that $J_{P}$ vanishes identically in restriction to the module $\mathfrak{P}(U)$. The Leibntiz property of the almost bracket $[., .]_{P}$ on $\mathfrak{P}(U)$ and relation (2.11) imply that the restriction of $P$ to $\mathfrak{P}(U)$ is a Lie algebra morphism. The proof of (2) will be complete by application of the following Lemma 2.3.7.

Lemma 2.3.7. Let $\left(T^{\prime} M, M, P,\{., .\}_{P}\right)$ be a partial Poisson structure.

For each $x \in M$, there exists an open neighbourhood $U$ of $x$ such that the vector space $\mathfrak{P}_{x}(U)=\left\{d_{x} f, f \in \mathcal{A}(U)\right\}$ is equal to the fibre $T_{x}^{\prime} M$. 
Proof. Fix some $x \in M$. Since it is a local problem, according to the assumptions of the definition of a partial Poisson manifold, there exists an open neighbourhood $U$ of $x \in M$ such that over $U$ the bundles $T M, T^{\prime} M$ and $T^{\prime} M$ are trivializable. Thus, without loss of generality, we may assume that $U$ is an open set of $\mathbb{M}$, so that $T M_{\mid U}=U \times \mathbb{M}, T^{\prime} M_{\mid U}=U \times \mathbb{M}^{\prime}, T^{\prime} M_{\mid U}=U \times \mathbb{E}$ and if $i: \mathbb{E} \rightarrow \mathbb{M}^{\prime}$ is the natural inclusion then $\iota_{\mid U}(x, u)=(x, i(u))$. In this situation, any section of $T^{\prime} M$ over $U$ is characterized by a smooth map $\alpha: U \rightarrow \mathbb{E} \subset \mathbb{M}^{\prime}$. Now, choose any $\alpha \in T_{x}^{\prime} M \subset T_{x}^{\prime} M$ and consider $f_{\alpha}: U \rightarrow \mathbb{R}$ defined by $f_{\alpha}(z)=\left\langle\alpha, z>\right.$. Then $d_{x} f_{\alpha}(u)=<\alpha, u>$ for all $u \in U$, so $f_{\alpha}$ belongs to $\mathcal{A}(U)$ since $d_{x}^{k} f_{\alpha}=0$ for $k \geq 2$ which ends the proof.

Remark 2.3.8. In finite dimension, if $T^{\prime} M=T^{*} M$ then $\mathcal{A}(M)=\mathcal{C}^{\infty}(M)$ and so $\mathcal{M}$ is exactly the module $\Lambda^{1}(M)$ of 1-forms on $M$. So we recover the classical result that, for a finite dimensional Poisson manifold, we obtain a Lie algebroid structure $\left(T^{*} M, M, P,[,]_{P}\right)$.

In the infinite dimensional case, even if $T^{\prime} M=T^{*} M$ and so $\mathcal{A}(M)=\mathcal{C}^{\infty}(M)$, this result is not true in general: we only get a partial Lie algebroid $\left(\mathcal{M}, M, P,[,]_{P}\right)$.

\subsection{Almost symplectic foliation associated to a partial Pois- son structure}

\subsubsection{Preliminaries and notations}

Let $M$ be a convenient manifold.

Definition 2.4.1. An almost symplectic (convenient) manifold is a pair $(M, \omega)$ where $\omega$ is a differential 2-form such that the morphism

$$
\omega^{b}: T M \rightarrow T^{*} M
$$

defined by $\omega^{b}(X)=\omega(X$,$) is injective.$

Recall that a weak symplectic manifold is an almost symplectic manifold $(M, \omega)$ such that $\omega$ is closed (cf. Example 2.2.12).

From now on, we fix an almost symplectic manifold $(M, \omega)$. According to Vai], we have:

Definition 2.4.2. A vector field $X$ is called a Hamiltonian vector field for the almost symplectic manifold $(M, \omega)$ if

$$
L_{X} \omega=0, \quad i_{X} \omega=-d f \text { for some } f \in \mathcal{C}^{\infty}(M)
$$

where $L_{X}$ is the Lie derivative and $i_{X}$ is the inner product of forms.

Note that a function $f \in \mathcal{C}^{\infty}(M)$ which satisfies this relation is defined up to a constant. Such a function is called a Hamiltonian function relative to 
$(M, \omega)$. The set $\mathcal{C}_{\omega}^{\infty}(M)$ of Hamiltonian functions relative to $(M, \omega)$ has an algebra structure. Moreover, since we have

$$
i_{[X, Y]}=L_{X} i_{Y}-i_{Y} L_{X}
$$

it follows that, if $X$ and $Y$ are two Hamiltonian fields, then

$$
i_{[X, Y]} \omega=-d \omega(X, Y)
$$

Therefore $[X, Y]$ is also a Hamiltonian field. It follows that $\mathcal{C}_{\omega}^{\infty}(M)$ can be endowed with a Lie Poisson bracket defined by

$$
\{f, g\}_{\omega}=\omega([X, Y])
$$

if $i_{X} \omega=-d f$ and $i_{Y} \omega=-d g$ (for more details, see [Vai]). If $\omega$ is closed, then we get the classical Lie Poisson bracket associated to a weak symplectic manifold (cf. Example 2.2.12). However when $\omega$ is not closed, the set $\mathcal{C}_{\omega}^{\infty}(M)$ is in general very small and it can be reduced to constant functions on $M$ (cf. VVai] for such examples).

\subsubsection{Almost symplectic foliation of a partial Poisson structure}

Definition 2.4.3. Let $M$ be a convenient manifold.

1. A distribution $\Delta$ on $M$ is an assignment $\Delta: x \mapsto \Delta_{x} \subset T_{x} M$ on $M$ where $\Delta_{x}$ is a subspace of $T_{x} M$, fibre of the dynamical tangent bundle $T M$ of $M$.

2. A vector field $X$ on $M$, defined on an open set Dom $(X)$, is called tangent to a distribution $\Delta$ if $X(x)$ belongs to $\Delta_{x}$ for all $x \in \operatorname{Dom}(X)$.

3. A distribution $\Delta$ on $M$ is called integrable if, for all $x \in M$, there exists a weak submanifold $L$ of $M$ such that $T_{x} L=\Delta_{x}$ for all $x \in L$. In this case, $L$ is called an integral manifold of $\Delta$ through $x$.

4. An integral manifold $L$ of a distribution $\Delta$ is called maximal if any integral manifold $L^{\prime}$ of $\Delta$ is an open submanifold of $L$.

If a distribution $\Delta$ is integrable, then the set $\mathcal{F}$ of maximal integral manifolds of $\Delta$ gives rise to a partition of $M$ called a foliation of $M$.

Let $\left(M, \mathcal{A}(M),\{,\}_{P}\right)$ be a partial Poisson manifold on $M$. The image $\Delta_{x}=$ $P\left(T_{x}^{\prime} M\right)$ gives rise to a smooth distribution $\Delta$ on $M$ called the characteristic distribution of the partial Poisson structure. Note that each Hamiltonian vector field $P(d f)$ of a function $f \in \mathcal{A}(M)$ is tangent to $\Delta$.

On $T^{\prime} M$, we have a natural skew-symmetric bilinear form $\Omega$ defined as follows: for any $\alpha$ and $\beta$ in $T_{x}^{\prime} M$, we have $\Omega(\alpha, \beta)=\{f, g\}$ if $f$ and $g$ are smooth functions defined on a neighbourhood of $x$ and such that $d f(x)=\alpha$ and $d g(x)=\beta$ (this 
definition is independent of the choice of $f$ and $g$ ). Note that from (2.3), we have

$$
\Omega(\alpha, \beta)=<\alpha, P(\beta)>=-<\beta, P(\alpha)>
$$

Now, according to (2.13), for each $x$, on the quotient $T_{x}^{\prime} M / \operatorname{ker} P_{x}$ we get a well defined skew-symmetric bilinear form $\hat{\Omega}_{x}$. On the other hand, let $\hat{P}_{x}$ : $T_{x}^{\prime} M / \operatorname{ker} P_{x} \rightarrow \Delta_{x}$ be the canonical isomorphism associated to $P_{x}$ between convenient spaces. In this way, we get a skew-symmetric bilinear form $\omega_{x}$ on $\Delta_{x}$ such that :

$$
\left[\hat{P}_{x}\right]^{*} \omega_{x}=\hat{\Omega}_{x}
$$

Moreover by construction $\left(\Delta_{x}, \omega_{x}\right)$ is a weak symplectic manifold. Therefore we introduce:

Definition 2.4.4. Let $\left(M, \mathcal{A}(M),\{,\}_{P}\right)$ be a partial Poisson structure on $M$.

1. An almost symplectic (resp. weak symplectic) leaf of $\Delta$ is a convenient manifold $L \subset M$ with the following properties :

(i) $L$ is an integral manifold of $\Delta$;

(ii) there exists a 2-form $\omega_{L}$ on $L$ such that $\left(L, \omega_{L}\right)$ is an almost symplectic (resp. weak symplectic) manifold such that $\left(\omega_{L}\right)_{x}=\omega_{x}$ for all $x \in L$.

2. Assume that the characteristic distribution $\Delta$ of the partial Poisson structure is integrable. If each maximal integral manifold is an almost symplectic (resp. weak symplectic) leaf, the associated foliation $\mathcal{F}$ is called an almost symplectic (resp. weak symplectic) foliation.

We then have the following result:

Proposition 2.4.5. If the characteristic distribution $\Delta$ of a partial Poisson structure $\left(M, \mathcal{A}(M),\{,\}_{P}\right)$ is integrable, then the associated foliation $\mathcal{F}$ is an almost symplectic foliation.

Proof. Fix some maximal leaf $L$ of the foliation defined by $\Delta=P\left(T^{\prime} M\right)$. We have already seen that, for any $x \in L$, we have a skew-symmetric bilinear form $\omega_{x}$ on $\Delta_{x}$. Therefore we must show that the field $x \mapsto \omega_{x}$ gives rise to an almost symplectic form $\omega_{L}$ on $L$. If $i: L \rightarrow M$ is the natural inclusion, then we can consider the pull back of $\tilde{p^{\prime}}: \widetilde{T^{\prime} M} \rightarrow L$ of $p^{\prime}: T^{\prime} M \rightarrow M$ and we have a morphism $\tilde{\imath}: \widetilde{T^{\prime} M} \rightarrow T^{\prime} M$ over $i$ which is an isomorphism between each fiber. Therefore the kernel of the morphism $\tilde{P}=P \circ \tilde{\imath}$ is a convenient subbundle of $\widetilde{T^{\prime} M}$. Since $P\left(T_{x}^{\prime} M\right)=T_{x} L$ for $x \in L$, we obtain a bundle isomorphism $\widehat{P}: \widetilde{T^{\prime} M} / \operatorname{ker} \tilde{P} \rightarrow T L$. Moreover $\tilde{\Omega}=\tilde{\imath}^{*} \Omega$ is a smooth skew-symmetric bilinear form on $\widetilde{T^{\prime} M}$ such that

$$
\tilde{\Omega}(\tilde{\alpha}, \tilde{\beta})=<\tilde{\imath}(\tilde{\alpha}), \tilde{P}(\tilde{\beta})>=-<\tilde{\imath}(\tilde{\beta}), \tilde{P}(\tilde{\alpha})>
$$


for all sections $\tilde{\alpha}$ and $\tilde{\beta}$ of $\widetilde{T^{\prime} M} \rightarrow L$. It follows that $\tilde{\Omega}$ induces on the quotient bundle $\widetilde{T^{\prime} M} / \operatorname{ker} \tilde{P}$ a smooth skew-symmetric bilinear form $\tilde{\omega}$. Moreover, $\omega_{L}=$ $\left(\widehat{P}^{-1}\right)^{*} \tilde{\omega}$ is a smooth skew-symmetric bilinear form on $L$ such that $\left(\omega_{L}\right)_{x}=\omega_{x}$ for all $x \in L$.

Remark 2.4.6. In the context of Proposition 2.4.5, on each leaf $L$ of $\mathcal{F}$, the associated 2-form is in general not closed. The Jacobi Identity satisfied by the Poisson bracket $\{,\}_{P}$ implies $d \omega_{L}(X, Y, Z)=0$, only for Hamiltonian vector fields $X, Y$ and $Z$ restricted to $L$. When $M$ is a finite dimensional manifold, then any vector field $X$ tangent to $L$ is a finite sum of type $\sum_{j \in J} \phi_{j} X_{f_{j}}$ where each $\phi_{j}$ is a function and $X_{f_{j}}$ is an hamiltonian vector field; so $\omega_{L}$ is closed as it is well known. But even in the context of Banach manifolds, the previous argument is no more true in general. However, when $T^{\prime} M=T^{*} M$, any form $\sigma \in T_{x}^{\prime} M$ can be written $\sigma=d_{x} f$ for some local smooth function $f$ around $x \in M$ and so, for $i \in\{1,2,3\}$, each $X_{i} \in T_{x} L$ can be written $X_{i}=P\left(d_{x} f_{i}\right)$ for some $f_{i}$ locally defined around $x$. It follows that, in this case, each leaf is a weak symplectic leaf.

\subsubsection{Existence of almost symplectic foliation for Poisson Banach manifolds}

We first recall some useful preliminaries which can be found in [Pel].

Let $\pi: E \rightarrow M$ be a Banach fiber bundle over $M$ with typical fiber $\mathbb{E}$, and let $\rho: E \rightarrow T M$ be a morphism of bundles whose kernel is supplemented in each fiber and whose range $\Delta_{x}=\operatorname{im} \rho_{x}$ is closed in $T_{x} M$ for all $x \in M$. We denote by $\widehat{\Gamma}(E)$ the set of local sections of $\pi: E \rightarrow M$, that is smooth maps $\sigma: U \subset M \rightarrow E$ such that $\pi \circ \sigma=I d_{U}$ where $U$ is an open set of $M$. The maximal open set of this type is called the domain of $\sigma$ and is denoted $\operatorname{Dom}(\sigma)$. A subset $\mathcal{S}$ of $\widehat{\Gamma}(E)$ is called a generating set if, for any $x \in M$, there exists a trivialization $\Theta: U \times \mathbb{E} \rightarrow E_{\mid U}$ of $E$ over an open set $U$ which contains $x$ such that $\mathcal{S}$ contains all local sections defined on $U$ of type $\hat{\alpha}: y \rightarrow \Theta(y, \alpha)$ for all $\alpha \in \mathbb{E}$.

We say that a generating set $\mathcal{S}$ satisfies the condition (LB) if:

for any local section $\sigma \in \mathcal{S}$, there exists an open set $V \subset \operatorname{Dom}(\sigma)$ and a trivialization $\Theta: U \times \mathbb{E} \rightarrow E_{\mid U}$ such that $\mathcal{S}$ contains all sections $\hat{\alpha}$ on $U$ for $\alpha \in \mathbb{E}$ and, for any $x \in V$, we have the following property: given any integral curve $\gamma:]-\varepsilon, \varepsilon[\rightarrow V$ of $X=\rho(\sigma)$ with $\gamma(0)=x$, there exists a smooth field $\Lambda:]-\varepsilon, \varepsilon[\rightarrow L(\mathbb{E}, \mathbb{E})$ such that

$$
\forall t \in]-\varepsilon, \varepsilon\left[, \forall \alpha \in \mathbb{E},[\rho(\sigma), \rho(\hat{\alpha})](\gamma(t))=\rho\left(\gamma(t), \Theta\left(\gamma(t), \Lambda_{t}(\alpha)\right)\right.\right.
$$

We then have the following result (cf. [Pel]).

Theorem 2.4.7. Let $(E, M, \rho)$ be a Banach anchored bundle such that the kernel of $\rho$ is supplemented in each fibre and whore range $\Delta=\operatorname{im} \rho$ is closed. 
Then $\Delta$ is an integrable distribution if and only there exists a generating set $\mathcal{S}$ which satisfies the condition (LB).

By application of this theorem, we obtain the following result of integrability for Banach anchored bundles.

Corollary 2.4.8. Let $(E, M, \rho)$ be a Banach anchored bundle such that the kernel of $\rho$ is supplemented in each fiber and whore range $\Delta=\operatorname{Im} \rho$ is closed. Assume that there exists a sheaf $\mathfrak{P}_{M}$ of $\mathcal{E}_{M}$-modules of sections of $T^{\prime} M$ such that $\left(E, M, \rho, \mathfrak{P}_{M}\right)$ is a strong partial Lie algebroid. Then $\Delta=\operatorname{Im} \rho$ is integrable.

Under the assumptions of Corollary 2.4.8 we have the following local result which is an easy adaptation of Proposition 2.13 in Pel].

Proposition 2.4.9. Let $x \in M$ and consider a local trivialization $\Theta: U \times \mathbb{E} \rightarrow$ $E_{\mid U}$ and a Banach subspace $\mathbb{S}$ such that $\mathbb{E} \equiv E_{x}=\operatorname{ker} \rho_{x} \oplus \mathbb{S}$. For any $u \in \mathbb{S}$, we denote by $X_{u}$ the vector field $X_{u}(x)=\rho \circ \Theta(x, u)$ and by $\phi_{t}^{X_{u}}$ the flow of $X_{u}$. Then we have:

1. Given any norm \|\| on $\mathbb{S}$, there exists a ball $B(0, r)$ in $\mathbb{S}$ such that $\phi_{t}^{X_{u}}$ is defined for all $t \in[0,1]$ and for all $u \in B(0, r)$.

2. If $\Phi: B \rightarrow M$ is the map defined by $\Phi(u)=\phi_{1}^{X_{u}}(x)$ for $u \in B \equiv B(0, r)$, there exists $\delta>0$ such that $\Phi: B(0, \delta) \rightarrow M$ is a weak injective closed immersion $M$.

3. For $\delta$ small enough, $\Phi(B(0, \delta))$ is an integral Banach manifold of $\Delta$ through $x$ modelled on the Banach space $\mathbb{S}$ provided with the initial norm \|\| .

Now let $\left(M, \mathcal{A}(M),\{,\}_{P}\right)$ be a partial Banach Poisson manifold. The following result is essentially an easy consequence of the previous sufficient condition of integrability.

Theorem 2.4.10. Let $\left(M, \mathcal{A}(M),\{,\}_{P}\right)$ be a partial Banach Poisson manifold such that the kernel of $P$ is supplemented in each fiber $T_{x}^{\prime} M$ of $T^{\prime} M$ and $P\left(T^{\prime} M\right)$ is a closed distribution. Then we have the following:

1. $\Delta=P\left(T^{\prime} M\right)$ is integrable and the foliation defined by $\Delta$ is an almost symplectic foliation;

2. On each maximal leaf $N$, if $\left(N, \omega_{N}\right)$ is the natural almost symplectic structure on $N$ (cf proof of Proposition 2.4.5), then the restriction $f_{N}$ of $f \in \mathcal{A}(M)$ belongs to $\mathcal{C}_{\omega_{N}}^{\infty}(M)$ and we have, for any $f$ and $g$ in $\mathcal{A}(M)$

$$
\left\{f_{\mid N}, g_{\mid N}\right\}_{P_{\mid N}}=\left\{f_{N}, g_{N}\right\}_{\omega_{N}} .
$$

Proof of Corollary 2.4.8. According to Theorem 2.4.7 we only have to prove that $\mathcal{S}=\mathfrak{P}_{M}$ satisfies the property (LB). For any smooth local section $\sigma$ : 
$U \rightarrow T^{\prime} M$, we set $Z_{\sigma}=P(\sigma)$. From our assumption, we have for any sections $\sigma_{1}, \sigma_{2} \in \mathcal{S}$ defined on $U$

$$
\left[Z \sigma_{1}, Z_{\sigma_{2}}\right]=\rho\left(\left[\sigma_{1}, \sigma_{2}\right]_{E}\right) .
$$

As (LB) is a local property, fix some $\sigma \in \mathcal{S}$ defined on an open set $U$ such that $\mathcal{S}$ contains all local sections defined on $U$ of type $\hat{\alpha}$ for all $\alpha \in \mathbb{E}$ and for an adequate trivialization $\Theta: U \times \mathbb{E} \rightarrow E_{\mid U}$. Such a choice of $U$ is is always true according to Lemma 2.3.7. Denote by $\phi_{t}^{Z_{\sigma}}$ the flow of $Z_{\sigma}$ defined on some open set $V \subset U$. Consider an integral curve $\gamma(t)=\phi_{t}^{Z_{\sigma}}(z)$ through $z \in V$ defined on ]$-\varepsilon, \varepsilon[$. For any $\alpha \in \mathbb{E}$, from (2.14), we have:

$$
\left.\left[Z_{\sigma}, Z_{\hat{\alpha}}\right](\gamma(t))=[\rho(\sigma), \rho(\hat{\alpha})](\gamma(t))=\rho\left([\sigma, \hat{\alpha}]_{E}\right)(\gamma(t))\right)
$$

Now, using the same arguments as the ones used in the proof of Lemma 3.11 of [Pel], we can show that the map

$$
y \mapsto\left(\alpha \mapsto[\sigma, \hat{\alpha}]_{E}(y)\right)
$$

is a smooth field of continuous endomorphisms of $\mathbb{E}$. It follows that $\mathcal{S}$ satisfies (LB), and then, $\Delta$ is integrable.

Proof of Theorem 2.4.10. From Proposition 2.3.6. all assumptions of Corollary 2.4 .8 are satisfied and so $\Delta$ is integrable.

The last other properties of the foliation defined by $\Delta$ are easy to prove and are left to the reader.

\section{Direct and inverse limit of partial Poisson Ba- nach manifolds}

\subsection{Direct and inverse limits of linear bundles}

We only recall the results obtained in the framework of direct limits in CabPel2. For the case of inverse limits, the reader can adapt the results obtained in [DGV].

Definition 3.1.1. A sequence $\left(E_{n}, \pi_{n}, M_{n}\right)_{n \in \mathbb{N}^{*}}$ of Banach vector bundles is called a strong ascending sequence of Banach vector bundles if the following assumptions are satisfied:

1. $\mathcal{M}=\left(M_{n}\right)_{n \in \mathbb{N}^{*}}$ is an ascending sequence of Banach $C^{\infty}$-manifolds, where $M_{n}$ is modelled on the Banach space $\mathbb{M}_{n}$ such that $\mathbb{M}_{n}$ is a supplemented Banach subspace of $\mathbb{M}_{n+1}$ and $\left(M_{n}, \varepsilon_{n}^{n+1}\right)$ is a weak submanifold of $M_{n+1}$;

2. The sequence $\left(E_{n}\right)_{n \in \mathbb{N}^{*}}$ is an ascending sequence such that the sequence of typical fibers $\left(\mathbb{E}_{n}\right)_{n \in \mathbb{N}^{*}}$ of $\left(E_{n}\right)_{n \in \mathbb{N}^{*}}$ is an ascending sequence of Banach spaces such that $\mathbb{E}_{n}$ is a supplemented Banach subspace of $\mathbb{E}_{n+1}$;

3. For each $n \in \mathbb{N}^{*}, \pi_{n+1} \circ \lambda_{n}^{n+1}=\varepsilon_{n}^{n+1} \circ \pi_{n}$ where $\lambda_{n}^{n+1}: E_{n} \longrightarrow E_{n+1}$ is the natural inclusion; 


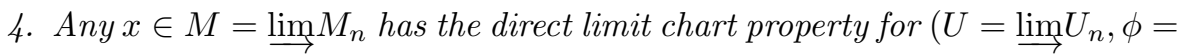
$\left.\underset{\lim _{\longrightarrow}}{ } \phi_{n}\right)$;

5. For each $n \in \mathbb{N}^{*}$, there exists a trivialization $\Psi_{n}:\left(\pi_{n}\right)^{-1}\left(U_{n}\right) \longrightarrow U_{n} \times \mathbb{E}_{n}$ such that the following diagram is commutative:

$$
\begin{array}{ccc}
\left(\pi_{n}\right)^{-1}\left(U_{n}\right) & \stackrel{\lambda_{n}^{n+1}}{\longrightarrow} & \left(\pi_{n+1}\right)^{-1}\left(U_{n+1}\right) \\
\Psi_{n} \downarrow & & \downarrow \Psi_{n+1} \\
U_{n} \times \mathbb{E}_{n} & \stackrel{\left(\varepsilon_{n}^{n+1} \times \iota_{n}^{n+1}\right)}{\longrightarrow} & U_{n+1} \times \mathbb{E}_{n+1} .
\end{array}
$$

We then have the following result (CabPel2, Proposition 41).

Proposition 3.1.2. Let $\left(E_{n}, \pi_{n}, M_{n}\right)_{n \in \mathbb{N}^{*}}$ be a strong ascending sequence of Banach vector bundles. We have:

1. $\lim _{n} E_{n}$ has a structure of non necessarily Hausdorff convenient manifold

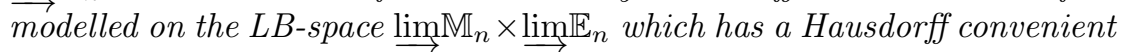
structure if and only if $\overrightarrow{M \text { is Hausdorff. }}$

2. $\left(\lim _{\longrightarrow} E_{n}, \stackrel{\lim \pi_{n}}{\longrightarrow}, \lim _{\longrightarrow} M_{n}\right)$ can be endowed with a structure of convenient vector bundle whose typical fiber is $\mathrm{lim}_{n}$ and whose structural group is the metrizable complete topological group $\mathbb{G}(\mathbb{E})$, projective limit of a sequence of Banach-Lie groups.

\subsection{Convenient Poisson morphisms}

Let $\varepsilon: M_{1} \rightarrow M_{2}$ be a smooth map between two convenient manifolds $M_{1}$ and $M_{2}$ modelled on $\mathbb{M}_{1}$ and $\mathbb{M}_{2}$ respectively.

We consider subbundles $T^{\prime} M_{1}$ and $T^{\mathrm{b}} M_{2}$ of $T^{*} M_{1}$ and $T^{*} M_{2}$ respectively, whose respective inclusion are convenient bundle morphisms and we denote by $\mathbb{F}_{1}$ and $\mathbb{F}_{2}$ respectively their typical fibres.

Let $T \varepsilon: T M_{1} \rightarrow T M_{2}$ be the tangent map of $\varepsilon$. The adjoint of the bounded operator $T_{x} \varepsilon: T_{x} M_{1} \rightarrow T_{\varepsilon(x)} M_{2}$ is denoted by $T_{\varepsilon(x)}^{*} \varepsilon: T_{\varepsilon(x)}^{*} M_{2} \rightarrow T_{x}^{*} M_{1}$ for all $x \in M$. If $\varepsilon$ is an injective weak immersion, we can define an adjoint bundle morphism, denoted $T^{*} \varepsilon$, from $\left\{T^{*} M_{2}\right\}_{\mid \varepsilon\left(M_{2}\right)}$ to $T^{*} M_{1}$ by:

$$
\left(T_{y^{*}}^{*} \varepsilon\right)\left(\alpha^{\prime}\right)=\alpha^{\prime} \circ T_{x} \varepsilon \text { if } \varepsilon(x)=y^{\prime}
$$

More generally, such a morphism does not exist, but, for each point $\varepsilon(x) \in$ $M_{2}$, the adjoint linear operator $T_{x}^{*} \varepsilon: T_{\varepsilon(x)}^{*} M_{2} \rightarrow T_{x}^{*} M_{1}$ is characterized by the equation (3.1) and so is a bounded linear operator. Moreover, in our context, the restriction of $T_{x}^{*} \varepsilon$ to $T_{\epsilon(x)}^{\prime} M_{2}$ is also a bounded linear operator from $T_{\epsilon(x)}^{\prime} M_{2}$ into $T_{x}^{*} M$ since the inclusion of $T_{\epsilon(x)}^{\prime} M_{2}$ in $T_{\epsilon(x)}^{*} M_{2}$ is bounded.

By abuse of notation, we will simply denote by $T^{*} \varepsilon$ the set of operators $T_{\varepsilon(x)}^{*} \varepsilon$ for $x \in M_{1}$. 
Remark 3.2.1. Assume that

$$
T_{x}^{*} \varepsilon\left(T_{\varepsilon(x)}^{\prime} M_{2}\right) \subset T_{x}^{\prime} M_{1} .
$$

Then the linear operator $T_{x}^{*} \varepsilon_{\mid T_{\varepsilon(x)}^{\prime} M_{2}}: T_{\varepsilon(x)}^{\prime} M_{2} \rightarrow T_{x}^{\prime} M_{1}$ is not bounded in general if $T_{x}^{\prime} M_{1}$ is provided with its own topology of convenient space. However, this operator is bounded if $T_{x}^{\prime} M_{1}$ is provided with the topology induced from the topology on $T_{x}^{*} M_{1}$. In particular, if $T_{x}^{\prime} M_{1}$ is closed in $T_{x}^{*} M_{1}$, then $T_{x}^{*} \varepsilon_{\mid T_{\varepsilon(x)}^{\prime}} M_{2}$ : $T_{\varepsilon(x)}^{\prime} M_{2} \rightarrow T_{x}^{\prime} M_{1}$ is bounded.

Definition 3.2.2. For $i \in\{1,2\}$, let $p_{i}^{\prime}: T^{\prime} M_{i} \rightarrow M_{i}$ be a convenient weak subbundle of $p_{i}^{*}: T^{*} M_{i} \rightarrow M_{i}$.

1. Such pairs $\left(T^{\prime} M_{i}, T^{*} M_{i}\right)_{i=1,2}$ are called partial structures if moreover $T^{\prime} M_{1}$ is closed in $T^{*} M_{1}$.

2. We will say that a smooth map $\varepsilon: M_{1} \rightarrow M_{2}$ is compatible with these partial structures if, for all $x \in M$, we have:

(CPS) $T_{x}^{*} \varepsilon\left(T_{\varepsilon(x)}^{\prime} M_{2}\right) \subset T_{x}^{\prime} M_{1}$.

This property will be often cut down by

$$
\left(T^{*} \varepsilon\right)\left(T^{\prime} M_{2}\right) \subset T^{\prime} M_{1} .
$$

3. For $i \in\{1,2\}$, let $P_{i}: T^{\prime} M_{i} \rightarrow T M_{i}$ be a Poisson anchor over $M_{i}$. We say that $\varepsilon$ is convenient Poisson morphism if $\varepsilon$ satisfies the condition (CPS) and the following condition of compatibility of Poisson structures:

(CPPS) $P_{2}(\varepsilon(x), \xi)=T \varepsilon \circ P_{1}\left(x, T_{x}^{*} \varepsilon(\xi)\right)$

for all $x \in M$ and $\xi \in T_{\varepsilon(x)}^{*} M_{2}$.

This relation will be often cut down by

$$
P_{2}=T \varepsilon \circ P_{1} \circ T^{*} \varepsilon .
$$

The following result gives relations between convenient Poisson morphisms and convenient Poisson maps (cf. Definition 2.2.8) :

Theorem 3.2.3. Consider partial Poisson structures $\left(T^{\prime} M_{i}, T M_{i}, P_{i},\{,\}_{P_{i}}\right)$ for $i \in\{1,2\}$ such that $T^{\prime} M_{1}$ is closed in $T^{*} M_{1}$ and $\varepsilon: M_{1} \rightarrow M_{2}$ is a smooth map.

1. If $\varepsilon$ is a convenient Poisson morphism, for any open set $U_{1}$ in $M_{1}$ and $U_{2}$ in $M_{2}$ such that $\varepsilon\left(U_{1}\right) \subset U_{2}$, the restriction of $\varepsilon$ to $U_{1}$ is a convenient Poisson map from $\left(U_{1}, \mathcal{A}\left(U_{1}\right),\{.,,\}_{P_{1}}\right)$ to $\left(M_{2}, \mathcal{A}_{2},\{.,,\}_{P_{2}}\right)$ 
2. If $\left(T_{y}^{\prime} M_{2}\right)^{a}$ is the annihilator of $\left(T_{y}^{\prime} M_{2}\right)$ assume that we have $\left(T_{y}^{\prime} M_{2}\right)^{a} \cap$ $T_{y} M_{2}=\{0\}$ for all $y \in \varepsilon\left(M_{1}\right)$. Then conversely, if for any open set $U_{1}$ in $M_{1}$ and $U_{2}$ in $M_{2}$ such that $\varepsilon\left(U_{1}\right) \subset U_{2}$, the restriction of $\varepsilon$ to $U_{1}$ is a convenient Poisson map from $\left(U_{1}, \mathcal{A}\left(U_{1}\right),\{.,,\}_{P_{1}}\right)$ to $\left(U_{2}, \mathcal{A}\left(U_{2}\right),\{.,,\}_{P_{2}}\right)$ then $\varepsilon$ is a convenient Poisson morphism.

\section{Remark 3.2.4.}

1. If $M_{1}$ and $M_{2}$ are finite dimensional Poisson manifolds, the assumption of Point (2) of Theorem 3.2.3 is always satisfied and, as it is well known, the assertions (1) and (2) are equivalent.

2. If $T^{\prime} M_{1}=T^{*} M_{1}$ and $P_{2}$ is partial symplectic then, we have a weak symplectic form $\Omega_{2}$ on $T M_{2}$ such that $P_{2}=\left(\Omega^{\prime}\right)^{-1}$ (cf. Example 2.2.10). As in [KriMic], 48, this implies that the canonical pairing $\langle.,$.$\rangle between$ $T^{\prime} M_{2}$ and $T M_{2}$ is non degenerate and thus the assumption of Point (2) in Theorem 3.2.3 are always satisfied and so the assertions (1) and (2) are equivalent.

3. In general, there will exist at least one point $y \in \varepsilon\left(M_{1}\right)$ at which $\left(T_{y}^{\prime} M_{2}\right)^{a} \cap$ $T_{y} M_{2} \neq\{0\}$ and so the assertions (1) and (2) are not equivalent.

For instance, it is always the case if $T^{\prime} M_{1}=T^{*} M_{1}, \mathbb{M}_{2}$ is reflexive, $\mathbb{F}_{2} \neq \mathbb{M}_{2}^{*}$ and not dense $\mathbb{M}_{2}^{*}$ and $\varepsilon$ is a submersion.

The proof of this Theorem needs some auxiliary results which will be developed now.

Consider a chart $(U, \phi)$ of a convenient manifold $M$ and $T \phi: T M_{\mid U}=$ $p_{M}^{-1}(U) \rightarrow \phi(U) \times \mathbb{M}$ the associated trivialization of $T M$. Then $T^{*} \phi: \phi(U) \times$ $\mathbb{M}^{\prime} \rightarrow T^{*} M_{\mid U}=\left(p_{M}^{*}\right)^{-1}(U)$ is a bundle isomorphism, and so its inverse morphism $T^{*} \phi^{-1}$ is a trivialization of $T^{*} M$ canonically associated to the chart $(U, \phi)$.

Assume that we have the property (3.3). For any $x \in M_{1}$, there exist charts $(U, \phi)$ around $x$ in $M_{1}$ and $\left(U_{2}, \phi_{2}\right)$ around $\varepsilon(x)$ in $M_{2}$ respectively such that:

1. The restriction $T^{*} \phi^{-1}$ and $T^{*} \phi^{\prime-1}$ to $\left\{T^{\prime} M_{1}\right\}_{\mid U_{1}}$ and $\left\{T^{\prime} M_{2}\right\}_{\mid U_{2}}$ are trivializations onto $\phi\left(U_{1}\right) \times \mathbb{F}_{1}$ and $\phi_{2}\left(U_{2}\right) \times \mathbb{F}_{2}$ respectively;

2. For all $z \in \phi(U)$, we have:

$$
\left.\left\{\left(T^{*}\left(\phi_{2}\right) \circ \varepsilon \circ \phi_{1}^{-1}\right)\left(\phi_{2}\right) \circ \varepsilon \circ \phi_{1}^{-1}(z)\right\} \times \mathbb{F}_{2}\right) \subset\{z\} \times \mathbb{F}_{1}
$$

Such charts $\left(U_{1}, \phi_{1}\right)$ and $\left(U_{2}, \phi_{2}\right)$ will be called compatible with this Property.

Proposition 3.2.5. Consider two partial structures $\left(T^{\prime} M_{i}, T^{*} M_{i}\right) i=1,2$ (cf Definition 3.2.2 Point 1.)

Given a smooth map $\varepsilon: M_{1} \rightarrow M_{2}$, we have 
$\varepsilon^{*}\left(\mathcal{A}\left(U_{2}\right)\right) \subset \mathcal{A}\left(U_{1}\right)$ for any open set $U_{1}$ of $M_{1}$ such that $\varepsilon\left(U_{1}\right)$ is contained in an open set $U_{2}$ of $M_{2}$ if and only if the property (CPS) is satisfied.

Sketch of the proof. According to Lemma 2.3.7 the first assertion of Proposition 3.2.5 implies clearly property (CPS). We have to prove the converse.

Suppose that property (CPS) is satisfied. Since the problem is local, and according to compatible charts as previously, we may assume that, for $i \in\{1,2\}$, $U_{i}$ is a $c^{\infty}$ open set in $\mathbb{M}_{i}$ such that $T M_{i}=U_{i} \times \mathbb{M}_{i}, T^{\prime} M_{i}=U_{i} \times \mathbb{F}_{i}$ and $\varepsilon$ is a smooth map from $U_{1} \rightarrow U_{2}$ where

$-T_{x} \varepsilon\left(\{x\} \times \mathbb{M}_{1}\right) \subset\{\varepsilon(x)\} \times \mathbb{M}_{2}$

$-T_{x}^{*} \varepsilon\left(\{\varepsilon(x)\} \times \mathbb{F}_{2}\right) \subset\{x\} \times \mathbb{F}_{1}$.

Moreover, by assumption, $\mathbb{F}_{1}$ is closed in $\mathbb{M}_{1}^{*}$.

Since $\varepsilon^{*}(f)=f \circ \varepsilon$, we have

$$
d_{x}(f \circ \varepsilon)=d_{\varepsilon(x)} f \circ T_{x} \varepsilon=T_{x}^{*} \varepsilon\left(d_{\varepsilon(x)} f\right)
$$

Since $f$ belongs to $\mathcal{A}\left(U_{2}\right)$, the map $d_{x} f$ belongs to $T_{y}^{\prime} M_{2}$. Thus, according to relation (3.3), the previous relation implies that $d_{x}(f \circ \varepsilon)$ belongs to $T_{x}^{\prime} M_{1}$ and is bounded since $\mathbb{F}_{1}$ is closed in $\mathbb{M}_{1}^{*}$.

As in the proof of Proposition 2.2.4 2., by induction, we can show that

$$
d_{x}^{k}(f \circ \varepsilon)\left(u_{1}, \ldots, u_{k}\right)
$$

is a summation of terms of type

$$
\left.d_{\varepsilon(x)}^{l} f\left(T_{x} \varepsilon\left(u^{\sigma^{1}}\right), T_{x}^{2} \varepsilon\left(u^{\sigma^{2}}\right), \ldots, T_{x}^{i} \varepsilon\left(u^{\sigma^{i}}\right), \ldots, T_{x}^{h} \varepsilon\left(u^{\sigma^{h}}\right)\right)\right)
$$

where

- the set $\left\{\sigma^{1}, \ldots, \sigma^{h}\right\}$ is a partition of the set $\{1, \ldots, k\}$;

- for $\sigma^{i}$, one has:

- each $\sigma^{i}$ is a disjoint union of $\nu_{i}$ strictly increasing sequence of length $i$, that is $\sigma_{j}^{i}:=\left(\left(s_{j}^{i}\right)_{1}, \ldots,\left(s_{j}^{i}\right)_{i}\right)$ if $\nu_{i} \geq 1$;

- otherwise $\sigma^{i}$ is an empty set

- for all $1 \leq l \leq k$ and $h+l=k$, we have

$$
\left\{\begin{aligned}
\nu_{1}+2 \nu_{2}+\cdots+i \nu_{i}+\cdots+h \nu_{h} & =k \\
\nu_{1}+\cdots+\nu_{i}+\cdots+\nu_{h} & =l
\end{aligned}\right.
$$

- If $\nu_{i} \geq 1$ and $\sigma_{j}^{i}=\left(\left(s_{j}^{i}\right)_{1}, \ldots,\left(s_{j}^{i}\right)_{i}\right)$, for $j \in\left\{1, \ldots, \nu_{i}\right\}$, we set

$$
\begin{aligned}
u_{\sigma_{j}^{i}} & =\left(u_{\left(s_{j}^{i}\right)_{1}}, \ldots, u_{\left(s_{j}^{i}\right)_{i}}\right) \\
T_{x}^{i} \varepsilon\left(u^{\sigma^{i}}\right) & =\left(T_{x}^{i} \varepsilon\left(u_{\sigma_{1}^{i}}\right) \ldots, T_{x}^{i} \varepsilon\left(u_{\sigma_{\nu_{i}}^{i}}\right)\right)
\end{aligned}
$$


Note that the term (3.5) is completely defined by such a partition $\left\{\sigma^{1}, \ldots, \sigma^{h}\right\}$ and the summation in the expression of $d_{x}^{k}(f \circ \varepsilon)\left(u_{1}, \ldots, u_{k}\right)$ is for all such partitions.

For the end of the proof we need the following Lemma:

Lemma 3.2.6. Let $\varepsilon$ be a smooth map from a $c^{\infty}$ open set $U$ around 0 in convenient space $E_{1}$ into a convenient space $E_{2}$.

Consider a convenient space $F_{2}$ contained in $E_{2}^{*}$ with bounded inclusion and a closed convenient subspace $F_{1}$ of $E_{1}^{*}$. If the restriction of $\left(T_{x} \varepsilon\right)^{*}$ to $F_{2}$ is a bounded linear map from $F_{2}$ to $F_{1}$ for all $x \in U$ and all $u_{2}, \ldots, u_{n} \in E_{1}$, then $\left(\left(T_{x}^{n} \varepsilon\right)\left(., u_{2}, \ldots, u_{n}\right)\right)^{*}$ is a bounded linear map from $F_{2}$ to $F_{1}$, for integer $n$ and $x \in U$.

According to this Lemma, we fix such a partition $\left\{\sigma^{1}, \ldots, \sigma^{h}\right\}$. Then 1 belongs to one and only one $\sigma_{j}^{i_{0}}:=\left(\left(s_{j}^{i_{0}}\right)_{1}, \ldots,\left(s_{j}^{i_{0}}\right)_{i_{0}}\right)$ and since this sequence is strictly increasing, we $\left(s_{j}^{i_{0}}\right)_{1}=1$. The corresponding term is

$$
T_{x}^{i_{0}} \varepsilon\left(u^{\sigma_{j}^{i_{0}}}\right)=T_{x}^{l} \varepsilon\left(u_{\left(s_{j}^{i_{0}}\right)_{1}}, \ldots, u_{\left(s_{j}^{i_{0}}\right)_{i_{0}}}\right)
$$

After deleting $u_{\left(s_{j}^{l}\right)_{1}}:=u_{1}$ in this term we obtain the bounded linear map

$$
\widehat{\varepsilon_{1}}: u_{1} \mapsto T_{x}^{i_{0}} \varepsilon\left(u_{1}, u_{\left(s_{j}^{i_{0}}\right)_{2}}, \ldots, u_{\left(s_{j}^{i_{0}}\right)_{i_{0}}}\right)
$$

By application of Lemma 3.2.6, the restriction of $\left(\widehat{\varepsilon_{1}}\right)^{*}$ to $\mathbb{F}_{2}$ is a bounded linear map into $\mathbb{F}_{1}$.

Associated to this partition, we consider again the corresponding term (3.5)

$$
d_{\varepsilon(x)}^{l} f\left(T_{x} \varepsilon\left(u^{\sigma^{1}}\right), T_{x}^{2} \varepsilon\left(u^{\sigma^{2}}\right), \ldots, T_{x}^{i} \varepsilon\left(u^{\sigma^{i}}\right), \ldots, T_{x}^{h} \varepsilon\left(u^{\sigma^{h}}\right)\right)
$$

In this expression, we have exactly $h$ non zero vectors

$$
T_{x} \varepsilon\left(u^{\sigma^{1}}\right), T_{x}^{2} \varepsilon\left(u^{\sigma^{2}}\right), \ldots, T_{x}^{i} \varepsilon\left(u^{\sigma^{i}}\right), \ldots, T_{x}^{h} \varepsilon\left(u^{\sigma^{h}}\right)
$$

which belong to $T_{\varepsilon(x)} M_{1} \equiv \mathbb{F}_{2}$ which we denote by $v_{1}, \ldots, v_{h}$. Among these vectors, we have one and only one which is equal to $\widehat{\varepsilon_{1}}\left(u_{1}\right)$ and we may assume that $v_{1}=\widehat{\varepsilon_{1}}\left(u_{1}\right)$;

Finally, after having deleted $u_{1}$ in (3.6), we obtain a term of type

$$
\widehat{\varepsilon}_{1}^{*}\left(d_{\varepsilon(x)}^{l} f\left(., v_{2}, \ldots, \ldots v_{h}\right)\right) .
$$

which belongs to $\mathbb{F}_{1}$ from the construction of $\left(\varepsilon_{1}\right)^{*}$. But such a property is true for any term of the decomposition of $d_{x}^{k}(f \circ \varepsilon)\left(., u_{2}, \ldots, u_{k}\right)$ for any $k \in \mathbb{N}$ and any $u_{2}, \ldots, u_{k}$ in $T_{x} M_{1}$. This implies that $d_{x}^{k}(f \circ \varepsilon)\left(., u_{2}, \ldots, u_{k}\right)$ belongs to $T_{x} M_{1}$ for any $k \in \mathbb{N}$ and any $u_{2}, \ldots, u_{k}$ in $T_{x} M_{1}$ and so the proof is complete, according to Proposition 2.2.4. 
Proof of Lemma 3.2.6. Fix some $x \in U$. By assumption the result is true for $n=1$. Assume that the result is true for all $1 \leq i<n$. Then according to the definition of $d_{x}^{n} \varepsilon$ (cf. KriMic, 5.11), we have

$$
T_{x}^{n} \varepsilon\left(u, u_{2}, \ldots, u_{n}\right)=\lim _{t \rightarrow 0}\left(\frac{1}{t}\left(T_{x+t u}^{n-1} \varepsilon\left(u_{2}, \ldots, u_{n}\right)-T_{x}^{n-1} \varepsilon\left(u_{2}, \ldots, u_{n}\right)\right)\right)
$$

On the other hand, for any $\alpha \in F_{2}$, we obtain

$$
\left(T_{x}^{n} \varepsilon\left(., u_{2}, \ldots, u_{n}\right)\right)^{*}(\alpha)=\alpha \circ\left(T_{x}^{n} \varepsilon\left(., u_{2}, \ldots, u_{n}\right)\right)
$$

Now the map

$$
t \mapsto \alpha \circ T_{x+t u}^{n-1} \varepsilon\left(., u_{2}, \ldots, u_{n}\right)
$$

is a smooth map from $E_{2}^{*}$ to $E_{1}^{*}$.

Now, since $\alpha$ is a bounded linear form, and so is smooth, by chain rules we have:

$$
\frac{d}{d t}_{\mid t=0}\left(\alpha \circ\left(T_{x+t u}^{n-1} \varepsilon\left(., u_{2}, \ldots, u_{n}\right)\right)\right)=\alpha \circ\left(T_{x}^{n} \varepsilon\left(., u, u_{2} \ldots, u_{n}\right)\right)
$$

But the inclusion of $F_{2}$ in $E_{2}$ is bounded, $F_{1}$ is closed in $E_{1}^{*}$ and the map (3.7) takes values in $F_{1}$ from the inductive assumption. This implies that $\alpha \circ$ $\left(T_{x}^{n} \varepsilon\left(., u, u_{2} \ldots, u_{n}\right)\right)$ belongs to $F_{1}$ for any $\alpha \in F_{2}$. But the map $\alpha \mapsto \alpha \circ$ $\left(T_{x}^{n} \varepsilon\left(., u, u_{2} \ldots, u_{n}\right)\right)$ is a bounded map from $F_{2}$ to $E_{1}^{*}$ which takes values in $F_{1}$. Since $F_{1}$ is closed it follows that this map is a linear bounded map from $F_{2}$ to $F_{1}$.

Proof of Theorem 3.2.3.

1. Assume that $\varepsilon$ is a convenient Poisson morphism.

Fix an open $U_{i}$ in $M_{i}$ such that $\varepsilon\left(U_{1}\right) \subset U_{2}$. Then according to (CPS) and Proposition 3.2 .5 we have

$$
\varepsilon^{*}\left(\mathcal{A}\left(U_{2}\right)\right) \subset \mathcal{A}\left(U_{1}\right)
$$

Fix some $x \in U_{1}$. Consider any local section $\alpha_{1}$ of $T^{\prime} M_{1}$ and $\alpha_{2}$ of $T^{\prime} M_{2}$ ) around $x$ and $\varepsilon(x)$ respectively, such that $\alpha_{2}(\varepsilon(x))=\left(T_{x} \varepsilon\right)^{*} \alpha_{1}(x)$. From condition (CPPS) and Lemma 2.3.7, we have:

$$
P_{2}\left(\alpha_{2}(\varepsilon(x))=T_{x} \varepsilon \circ P_{1}\left(\alpha_{1}(x)\right)\right.
$$

So if for $i=1,2, \beta_{i}$ are also a section of $T^{\prime} M_{i}$ such that $\beta_{2}(\varepsilon(x))=\left(T_{x} \varepsilon\right)^{*} \beta_{1}(x)$, we will have

$$
<\beta_{2}, P_{2}\left(\alpha_{2}\right)>\varepsilon(x)=<\left(T_{x} \varepsilon\right)^{*}\left(\beta_{1}(x)\right), T_{x} \varepsilon \circ P_{1}\left(\alpha_{1}(x)\right)
$$

But if $f$ and $g$ in $\mathcal{E}\left(U_{2}\right)$, we have:

$$
\{f, g\}_{P_{2}}=-<d f, P_{2}(d g)>
$$

For $\alpha_{1}(x)=d(g \circ \varepsilon)(x)=\left(T_{x} \varepsilon\right)^{*} d g(x)$ and $\beta_{1}(x)=d(f \circ \varepsilon)(x)=\left(T_{x} \varepsilon\right)^{*} d f(x)$ in (3.8) we get the announced result at point $x$ according to (3.8). Since this true 
for any $x \in U$, this ends the proof.

2. We consider the assumptions of Point (2).

Fix some point $x \in M_{1}$ and let $U_{2}$ be an open set around $\varepsilon(x)$ in $M_{2}$ such that set $\left\{d_{\varepsilon(x)} f, f \in \mathcal{E}\left(U_{2}\right)\right\}$ is equal to $T_{\varepsilon(x)}^{\prime} M_{2}$ (cf. Lemma 2.3.7). Since $d_{x}(f \circ \varepsilon)=\left(T_{x}^{*} \varepsilon\right)(d f)$, it follows that $d_{x}(f \circ \varepsilon)$ belongs to $\left(T_{x}^{*} \varepsilon\right)\left(T_{\varepsilon(x)}^{\prime} M_{2}\right)$ and so the set $\left.\left\{d_{x}(f \circ \varepsilon)\right), f \in \mathcal{E}\left(U_{2}\right)\right\}$ generates $\left(T_{x}^{*} \varepsilon\right)\left(T_{\varepsilon(x)}^{\prime} M_{2}\right)$. Thus condition (CPS) is satisfied.

According to the definition of the Poisson bracket from a Poisson anchor and Poisson map we have

$$
<d_{\varepsilon(x)} g, T_{x} \varepsilon \circ P_{1} \circ\left(T_{x}^{*} \varepsilon\right)\left(d_{\varepsilon(x)} f\right)>=<d_{\varepsilon(x)} g, P_{2}\left(d_{\varepsilon(x)} f\right)>
$$

for all $f, g \in \mathcal{A}\left(U_{2}\right)$. Since the set $\left\{d_{\varepsilon(x)} g, g \in \mathcal{A}\left(U_{2}\right)\right\}$ generates $T_{\varepsilon(x)}^{\prime} M_{2}$, this implies that that the range of $T_{x} \varepsilon \circ P_{1} \circ\left(T_{x}^{*} \varepsilon\right)\left(d_{\varepsilon(x)} f\right)-P_{2}\left(d_{\varepsilon(x)} f\right)$ is contained in $\left(T_{\varepsilon(x)}^{\prime} M_{2}\right)^{a} \cap T_{\varepsilon(x)} M_{2}$. But by assumption this vector space is reduced to $\{0\}$. This implies that the condition (CPPS) is also satisfied, which ends the proof.

Remark 3.2.7. When $\varepsilon$ is a Poisson map between two partial Poisson manifolds $\left(M_{1}, \mathcal{A}_{1},\{., .\}_{P_{1}}\right)$ and $\left(M_{2}, \mathcal{A}_{2},\{., .\}_{P_{2}}\right)$, then we have the following relation between Hamiltonian fields:

$$
X_{\varepsilon^{*} f}=T \varepsilon\left(X_{f}\right)
$$

\subsection{Partial Poisson structures on direct and inverse limits}

In this section $\left\{\left(M_{i}, C_{P_{i}}^{\infty}\left(M_{i}\right),\{,\}_{P_{i}}\right)\right\}_{i \in \mathbb{N}}$ is a sequence of partial Poisson Banach manifolds where $p_{i}^{\prime}: T^{\prime} M_{i} \rightarrow M_{i}$ is a Banach subbundle of $p_{M_{i}}^{*}: T^{*} M_{i} \rightarrow$ $M_{i}$ and $P_{i}: T^{\prime} M_{i} \rightarrow T M_{i}$ is a skew-symmetric morphism. We denote by $\mathbb{M}_{i}$ the Banach space on which $M_{i}$ is modelled, and by $\mathbb{F}_{i}$ the model of the typical fiber of $p_{i}^{\prime}: T^{\prime} M_{i} \rightarrow M_{i}$ and we assume that $\mathbb{F}_{i}$ is a Banach subspace of the dual $\mathbb{M}_{i}^{*}$ of $\mathbb{M}_{i}$.

\section{Definition 3.3.1.}

1. The sequence $\left\{\left(M_{i}, \mathcal{A}\left(M_{i}\right),\{,\}_{P_{i}}\right)\right\}_{i \in \mathbb{N}}$ is called a direct sequence of partial Poisson Banach manifolds if $\left(M_{i}\right)_{i \in \mathbb{N}^{*}}$ is an ascending sequence of Banach $C^{\infty}$-manifolds, where $M_{i}$ is modelled on the Banach space $\mathbb{M}_{i}$ such that $\mathbb{M}_{i}$ is a supplemented Banach subspace of $\mathbb{M}_{i+1}$ and such that $\left(M_{i}, \varepsilon_{i}\right.$ is a weak submanifold of $M_{i+1}$, and, for all $i \in \mathbb{N}$, we have the following properties:

(i) $T^{*} \epsilon_{i}\left(T^{\prime} M_{i+1}\right) \subset T^{\prime} M_{i}$;

(ii) $P_{i+1}=T \epsilon_{i} \circ P_{i} \circ T^{*} \epsilon_{i}$; 
(iii) Around each $x \in M$, there exists a sequence of charts $\left\{\left(U_{i}, \phi_{i}\right)\right\}_{i \in \mathbb{N}}$ such that

$\left(U=\lim _{\longrightarrow}\left(U_{i}\right), \phi=\lim _{\longrightarrow}\left(\phi_{i}\right)\right)$ is a chart of $x$ in $M$, so that the charts $\left(U_{i}, \phi_{i}\right)$ and $\left(U_{i+1}, \overrightarrow{\phi_{i+1}}\right)$ are compatible with Property (i).

2. The sequence $\left\{\left(M_{i}, \mathcal{A}\left(M_{i}\right),\{,\}_{P_{i}}\right)\right\}_{i \in \mathbb{N}}$ is called an inverse sequence of partial Poisson Banach manifolds if there exist submersions $\tau_{i}: M_{i+1} \rightarrow$ $M_{i}$ such that $\left\{\left(M_{i}, \tau_{i}\right)\right\}_{i \in \mathbb{N}}$ is a strong projective sequence of Banach manifolds fulfilling the following properties:

(i) $T^{*} \tau_{i}\left(T^{\prime} M_{i}\right) \subset T^{\prime} M_{i+1}$;

(ii) $P_{i}=T \tau_{i} \circ P_{i+1} \circ T^{*} \tau_{i}$;

(iii) Around each $x \in M$ there exists a sequence of charts $\left\{\left(U_{i}, \phi_{i}\right)\right\}_{i \in \mathbb{N}}$ such that

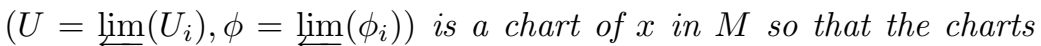
$\left(U_{i+1}, \phi_{i+1}\right)$ and $\left(U_{i}, \phi_{i}\right)$ are compatible with Property (i).

Remark 3.3.2. In the case (1), since $\left\{\left(M_{i}, \epsilon_{i}\right)\right\}_{i \in \mathbb{N}}$ is a direct set of Banach manifolds, we may assume that $\mathbb{M}_{i}$ is a Banach subspace of $\mathbb{M}_{i+1}$. We denote by $\hat{\epsilon}_{i}$ the natural inclusion of $\mathbb{M}_{i}$ in $\mathbb{M}_{i+1}$ and $\hat{\epsilon}_{i}^{*}: \mathbb{M}_{i+1}^{*} \rightarrow \mathbb{M}_{i}^{*}$ the adjoint operator. Note that $\epsilon_{i}^{*}$ is surjective. Since $\mathbb{F}_{i}$ is the typical fiber of $T^{\prime} M_{i}$, according to assumptions (i) and (iii) (compatibility with trivializations), we must have $\hat{\epsilon}_{i}^{*}\left(\mathbb{F}_{i+1}\right) \subset \mathbb{F}_{i}$. Moreover, since $\mathbb{F}_{i+1}$ is a Banach subspace of $\mathbb{M}_{i+1}^{*}$, then $\hat{\epsilon}_{i}^{*}\left(\mathbb{F}_{i+1}\right)$ is a Banach subspace of $\mathbb{F}_{i}$.

In the case (2), since $\left(M_{i}, \tau_{i}\right)_{i \in \mathbb{N}}$ is a strong projective set of Banach manifolds such that $\tau_{i}: M_{i+1} \rightarrow M_{i}$ is a submersion, there exists a surjective operator $\hat{\tau}_{i}$ from $\mathbb{M}_{i+1}$ onto $\mathbb{M}_{i}$. We denote by $\hat{\tau}_{i}^{*}: \mathbb{M}_{i}^{*} \rightarrow \mathbb{M}_{i+1}^{*}$ the adjoint operator which is injective. Note that $\hat{\tau}_{i}^{*}\left(\mathbb{M}_{i}^{*}\right)$ is a Banach subspace of $\mathbb{M}_{i+1}^{*}$. Again, since $\mathbb{F}_{i}$ is the typical fiber of $T^{\prime} M_{i}$, according to assumptions (i) and (iii), we must have $\hat{\tau}_{i}^{*}\left(\mathbb{F}_{i}\right) \subset \mathbb{F}_{i+1}$. Moreover, since $\mathbb{F}_{i}$ is a Banach subspace of $\mathbb{M}_{i}^{*}$, then $\hat{\tau}_{i}^{*}\left(\mathbb{F}_{i}\right)$ is a Banach subspace of $\mathbb{F}_{i+1}$ for all $i \in \mathbb{N}$.

Remark 3.3.3. Since by assumption, $p_{i}^{\prime}: T^{\prime} M_{i} \rightarrow M_{i}$ is a Banach subbundle of $p_{M_{i}}^{*}: T^{*} M_{i} \rightarrow M_{i}$ for all $i \in \mathbb{N}$, from property (i) and (ii) in Point (1) (resp. (2)) in Definition 3.3.1, the assumptions of Theorem 3.2.3 Point (1) are satisfied. It follows that $\epsilon_{i}$ (resp. $\tau_{i}$ ) is a Poisson morphism and so a Poisson map. It is easy to see that, for $j>i$, the same is true for $\epsilon_{i j}=\epsilon_{j-1} \circ \cdots \circ \epsilon_{i}: M_{i} \rightarrow M_{j}$ (resp. $\left.\tau_{i j}=\tau_{i} \circ \cdots \circ \tau_{j-1}: M_{j} \rightarrow M_{i}\right)$.

According to the previous definitions, we have the following result:

Theorem 3.3.4. Let $\left\{\left(M_{i}, \mathcal{A}\left(M_{i}\right),\{,\}_{P_{i}}\right)\right\}_{i \in \mathbb{N}}$ be a direct sequence (resp. an inverse sequence) of partial Poisson Banach manifolds and $M=\underline{\lim }\left(M_{i}\right)$ (resp. $\quad M=\lim \left(M_{i}\right)$ ). There exists a weak subbundle $p^{\prime}: T^{\prime} M \rightarrow M$ of $p_{M}^{*}: T^{*} M \rightarrow M$ and a skew-symmetric morphism $P: T^{\prime} M \rightarrow T M$ such 
that $\left(M, \mathcal{A}_{P}(M),\{,\}_{P}\right)$ is a partial Poisson structure on $M$ with the following characterization:

$$
\mathcal{A}_{P}(M)=\underset{\lim }{\longrightarrow} \mathcal{A}\left(M_{i}\right) \quad\left(\text { resp. } \mathcal{A}_{P}(M)=\lim _{\longleftarrow} \mathcal{A}\left(M_{i}\right)\right) ;
$$

if $\bar{\epsilon}_{i}: M_{i} \rightarrow M$ (resp. $\bar{\tau}_{i}: M \rightarrow M_{i}$ ) is the canonical injection (resp. projection) then $\bar{\epsilon}_{i}$ (resp. $\bar{\tau}_{i}$ ) is a Poisson map from $\left.\left(M_{i}, \mathcal{A}\left(M_{i}\right),\{,\}\right\}_{P_{i}}\right)$ to $\left(M, \mathcal{A}_{P}(M),\{,\}_{P}\right)$ (resp. from $\left(M, \mathcal{A}_{P}(M),\{,\}_{P}\right)$ to $\left(M_{i}, \mathcal{A}\left(M_{i}\right),\{,\}_{P_{i}}\right)$ ) for all $i \in \mathbb{N}$, and we have

$$
\{,\}_{P}=\underset{\lim }{\longrightarrow}\left(\{,\}_{P_{i}} \text { (resp. }\{,\}_{P}=\lim _{\longleftarrow}\left(\{,\}_{P_{i}}\right) .\right.
$$

\subsection{Proof of Theorem 3.3.4 in the case of direct limit}

Let $\left\{\left(M_{i}, \mathcal{A}\left(M_{i}\right),\{,\}_{P_{i}}\right)\right\}_{n \in \mathbb{N}}$ be a direct sequence of partial Poisson Banach manifolds. From the assumption in Definition 3.3.1 (1) and according to CabPel2, the direct limit $M=\underline{\lim }\left(M_{i}\right)$, is a convenient manifold.

Without loss of generality, we can assume that $M_{i} \subset M_{i+1}$; so $M=\bigcup_{i \in \mathbb{N}} M_{i}$ and $\epsilon_{i}$ is the natural inclusion of $M_{i}$ in $M_{i+1}$. For $j>i$, we denote by $\epsilon_{j i}=\epsilon_{j-1} \circ \cdots \circ \epsilon_{i}: M_{i} \rightarrow M_{j}$ the natural inclusion. Given a point $x \in M$, there exists $i \in \mathbb{N}$ such that $x$ belongs to $M_{i}$; let $n$ be the smallest of such integers $i$. On the one hand, $T_{x} \epsilon_{k n}: T_{x} M_{n} \rightarrow T_{x} M_{k}$ is an injective continuous linear map for all $k>n$. Since each $T_{x} M_{k}$ is isomorphic to the Banach space $\mathbb{M}_{k}$ for $k \geq n$, the set $\left\{\left(T_{x} M_{k}, T_{x} \epsilon_{k n}\right)\right\}_{k \geq n}$ is an ascending sequence of Banach space whose direct limit $T_{x} M=\underset{\lim }{\longrightarrow}\left(T_{x} M_{k}\right)=\bigcup_{k \geq n} T_{x} M_{k}$ is a convenient space. We set $T M=\bigcup_{x \in M} T_{x} M$. Let $p: T M \rightarrow M$ be the canonical projection.

Now, from Definition 3.3.1 (1), it follows that $T_{x}^{*} \epsilon_{k n}\left(T_{x}^{\prime} M_{k}\right)$ is a subset of $T_{x}^{\prime} M_{n}$ for all $k>n$. Therefore the $\left\{\left(T_{x}^{\prime} M_{k}, T_{x}^{*} \epsilon_{k n}\right)\right\}_{k>n}$ is an inverse sequence of Banach spaces. We set $T_{x}^{\prime} M=\lim \left(T_{x}^{\prime} M_{k}\right)$. In the same way, we can define the "projective dual" $T_{x}^{*} M=\lim \left(\overleftarrow{T_{x}^{*}} M_{k}\right)$ of $T_{x} M$. Of course, we have $T_{x}^{\prime} M \subset T_{x}^{*} M$ and these vector spaces provided with the inverse limit topology are Fréchet vector spaces. We set $T^{\prime} M=\bigcup_{x \in M} T_{x}^{\prime} M$ and $T^{*} M=\bigcup_{x \in M} T_{x}^{*} M$. We have canonical projections $p^{\prime}: T^{\prime} M \rightarrow M$ and $p^{*}: T^{*} M \rightarrow M$. We then have:

\section{Proposition 3.4.1.}

1. $p: T M \rightarrow M$ is a convenient bundle which is the kinematic bundle of $M$.

2. $p^{*}: T^{*} M \rightarrow M$ and $p^{\prime}: T^{\prime} M \rightarrow M$ are Fréchet locally trivial bundles over $M$. Moreover $p^{*}: T^{*} M \rightarrow M$ is the kinematic dual bundle of $M$.

3. There exists a canonical bundle morphism $P: T^{\prime} M \rightarrow T M$ characterized by

$$
P(x, \xi)=P\left(x, \lim _{\longleftarrow}{ }_{k i}\left(\xi_{k}\right)\right)=\underline{\lim }_{k \geq i}\left(P_{k}\left(x, \xi_{k}\right)\right) \text { if } x \in M_{i}
$$


Moreover, $P$ is skew-symmetric, relatively to the canonical dual pairing between $T^{*} M$ and $T M$ in restriction to $T^{\prime} M \times T M$.

Proof of Proposition 3.4.1. . We fix some $x \in M$ and assume that $x$ belongs to $M_{n}$ where $n$ is the smallest integer for which this property is true. Since $\left\{M_{i}, \epsilon_{i}\right\}_{i \in \mathbb{N}}$ has the limit chart property, there exists a chart $\left(U=\varliminf_{k>n}\left(U_{k}\right), \phi=\right.$ $\left.\lim _{k>n}\left(\phi_{k}\right)\right)$ around $x$ such that $\left(U_{k}, \phi_{k}\right)$ is a chart around $\epsilon_{k n}\left(x_{n}\right)$ in $M_{k}$. After restricting $U_{n}$ if necessary, we may assume that $U \cap M_{i}=\emptyset$ for $i<n$.

Proof of Point (1)

cf. CabPel2, proof of Proposition 41, 2.

Proof of Point (2)

We begin by considering $T_{M_{i}}^{*} M=\bigcup_{x \in M_{i}} T_{x}^{*} M$ and $T_{M_{i}}^{\prime} M=\bigcup_{x \in M_{i}} T_{x}^{\prime} M$ and we denote by $p_{M_{i}}^{*}: T_{M_{i}}^{*} M \rightarrow M_{i}$ and $p_{M_{i}}^{\prime}: T_{M_{i}}^{\prime} M \rightarrow M_{i}$ the canonical associate projections respectively. For each $i \in \mathbb{N}$ and $j \in \mathbb{N}$ such that $j>i$, we set $T_{M_{i}} M_{j}=\left(T M_{j}\right)_{\mid M_{i}}, T_{M_{i}}^{*} M_{j}=\left(T^{*} M_{j}\right)_{\mid M_{i}}$ and $\epsilon_{j i}=\epsilon_{j-1} \circ \cdots \circ \epsilon_{i}$. Then $T \epsilon_{j i}$ is a morphism from $T M_{i}$ to $T M_{j}$ and so we get a surjective morphism $T^{*} \epsilon_{j i}$ from $T_{M_{i}}^{*} M_{j}$ into $T^{*} M_{i}$ given by $T_{x_{i}}^{*} \epsilon_{j i}(\xi)=\xi_{\mid T_{x_{i}} M_{i}}$.

Thanks to the compatibilty relations of local charts, we have

$$
T^{*} \epsilon_{j i} \circ T^{*} \phi_{j}=T^{*} \phi_{i} \circ T^{*} \hat{\epsilon}_{j i} \text { on } \hat{\epsilon}_{j i} \circ \phi_{i}\left(U_{i}\right) \times \mathbb{M}_{j}^{*} .
$$

But $T^{*} \hat{\epsilon}_{j i}$ from $\mathbb{M}_{i} \times \mathbb{M}_{j}^{*}$ onto $\mathbb{M}_{i} \times \mathbb{M}_{i}^{*}$ is the map $(y, \xi) \mapsto\left(y, \xi_{\mid \mathbb{M}_{i}}\right)$. It follows that $\left\{\left(T_{M_{i}}^{*} M_{j}, T^{*} \epsilon_{j i}\right)\right\}_{j>i}$ is a strong projective sequence of bundles over $M_{i}$. From Gal, it follows that

$$
T_{M_{i}}^{*} M=\lim _{\longleftarrow}\left(T_{M_{i}}^{*} M_{j}\right) \rightarrow M_{i}
$$

is a Fréchet bundle. Note that $T_{M_{i}}^{*} M$ is a Fréchet manifold modelled on $\mathbb{M}_{i} \times$ $\lim _{j \geq i}\left(\mathbb{M}_{j}^{*}\right)$. According to Properties (i) and (iii) of Definition 3.3.1 the same arguments implies that $\left\{\left(T_{M_{i}}^{\prime} M_{j}, T^{*} \epsilon_{j i} \mid T_{M_{i}}^{\prime} M_{j}\right)\right\}_{j>i}$ is also a strong projective sequence of bundles over $M_{i}$.

$$
T_{M_{i}}^{\prime} M=\varliminf_{\longleftrightarrow}\left(T_{M_{i}}^{\prime} M_{j}\right) \rightarrow M_{i}
$$

is then a Fréchet bundle and a Fréchet manifold modelled on $\mathbb{M}_{i} \times \lim _{j>i}\left(\mathbb{F}_{j}\right)$.

On the one hand, $\epsilon_{j i}: M_{i} \rightarrow M_{j}$ is the natural inclusion, which induces the natural inclusion $\bar{\epsilon}_{j i}: T_{M_{i}}^{*} M \rightarrow T_{M_{j}}^{*} M$, namely $\bar{\epsilon}_{j i}(\omega)\left(x_{i}\right)=\omega\left(\epsilon_{j i}\left(x_{i}\right)\right)$ for any $x_{i} \in M_{i}$. Therefore $\left\{\left(T_{M_{j}}^{*} M, M_{j}, \bar{\epsilon}_{j i}\right)\right\}_{j>i}$ is a direct sequence of Fréchet bundles and so we have $T^{*} M=\lim \left(T_{M_{i}}^{*} M\right)$.

If we set $T_{U_{i}}^{*} M=T_{M_{i}}^{*} M_{\mid U_{i}}$, in the same way, we also have $T_{U}^{*} M=T_{M_{i}}^{*} M_{\mid U_{i}}=$ $\underset{\lim }{\longrightarrow}\left(T_{U_{i}}^{*} M_{j}\right)$

We have $\phi_{j i}=\phi_{j \mid U_{i}}=\phi_{i}$ and so $\phi_{j i}\left(U_{i}\right)=\phi_{i}\left(U_{i}\right)$. Therefore $T^{*} \phi_{j i}^{-1}$ is a trivialization of $T_{U_{i}}^{*} M_{j}$ onto $\phi_{i}\left(U_{i}\right) \times \mathbb{M}_{j}^{*}$ which is the restriction of $T^{*} \phi_{j}$ to $T_{U_{i}} M_{j}$. According to (3.10), and the results of Gal, we get a trivialization $\lim _{\longrightarrow}\left(T^{*} \phi_{j i}^{-1}\right)$ of $T_{U_{i}} M$ onto $\phi_{i}\left(U_{i}\right) \times \mathbb{M}^{*}$. Note that $\lim _{(}\left(T^{*} \phi_{j i}^{-1}\right)$ is in fact the 
adjoint operator of $\left.T \phi_{j}^{-1}\right)_{\mid\left\{\phi_{i}\left(x_{i}\right)\right\} \times \mathbb{M}^{*}}$ for all $x_{i} \in U_{i}$ where $\phi=\underline{\lim }\left(\phi_{j}\right)$ and so $T^{*} \phi_{\mid U_{i}}^{-1}=\underline{\lim _{\longrightarrow}}\left(T^{*} \phi_{j i}^{-1}\right)$

But since $\left(U=\underline{\lim }\left(U_{j}\right), \phi=\lim _{\longrightarrow}\left(\phi_{j}\right)\right)$ is a limit chart, for such fixed $i$, we have $U=\bigcup_{j \geq i} U_{j}$ and and then $T^{*} \phi_{j}{ }^{-1} \mid U_{i}=\varliminf_{j \geq i}\left(T^{*} \phi_{j i}{ }^{-1}\right)$ is a trivialization of $T^{*} M$ onto $\phi(U) \times \mathbb{M}^{*}$.

Thus we get a direct sequence of charts for the direct limit

$$
\left(T_{U_{i}}^{*} M=\underset{\longrightarrow}{\lim }\left(T_{U_{i}}^{*} M\right), T^{*} \phi^{-1}{ }_{\mid U_{i}}\right)=\underline{\lim }\left(T^{*} \phi_{j i}^{-1}\right)
$$

for the sequence $\left\{T_{M_{i}}^{*} M, \bar{\epsilon}_{j i}\right\}_{j>i}$ around any point $x \in M$ which belongs to $M_{i}$. Note that each manifold $T_{M_{i}}^{*} M$ is a closed immersed submanifold of $T_{M_{j}}^{*} M$ modelled on the Fréchet spaces $\mathbb{M}_{i} \times \lim _{l \geq i}\left(\mathbb{M}_{l}^{*}\right)$ and $\mathbb{M}_{j} \times \lim _{l \geq j}\left(\mathbb{M}_{l}^{*}\right)$ respectively and the first one is a closed Fréchet subspace of the second one. Note also that $\lim _{j \geq i}\left(\mathbb{M}_{j}\right) \times \lim _{l \geq i}\left(\mathbb{M}_{l}^{*}\right)$ is a convenient space which is diffeomorphic to $\mathbb{M} \times \mathbb{M}^{*}$ where $\mathbb{M}=\lim \left(\mathbb{M}_{j}\right)$. By same arguments as in CabPel2 Proposition 41 , we can prove that $\vec{T}^{*} M$ is a convenient manifold whose structural group is a metrizable complete topological group.

According to the assumption (ii) of Point (1) in Definition 3.3.1 the arguments used to prove that $T^{*} M=\underline{\lim }\left(T_{M_{i}}^{*} M\right)$ is a convenient bundle over $M$ (with typical fiber $\mathbb{F}=\lim _{\longleftarrow}\left(\mathbb{F}_{l}\right)$ ) still work for $T^{\prime} M=\underline{\lim }\left(T_{M_{i}}^{\prime} M\right)$.

Proof of Point (3)

Fix some $i \in \mathbb{N}$. According to assumption (ii) of Point (1) in Definition 3.3.1. $l \geq j \geq i$ by composition we have over $M_{l}$

$$
P_{l}=T \epsilon_{l j} \circ P_{j} \circ T^{*} \epsilon_{l j}: T_{M_{j}}^{\prime} M_{l} \rightarrow T_{M_{j}} M_{l}
$$

Therefore this relation is also true in restriction to $T_{M_{i}} M_{j}$ and, in this case, over $M_{i}$, we have the composition

$$
P_{l}=T \epsilon_{l i} \circ P_{j} \circ T^{*} \epsilon_{l i}: T_{M_{i}}^{\prime} M_{j} \rightarrow T_{M_{i}} M_{l}
$$

We set $P_{l j i}=T \epsilon_{l i} \circ P_{j_{\mid M_{i}}}$. Therefore we have a morphism $P_{l j i}: T_{M_{i}}^{\prime} M_{j} \rightarrow$ $T_{M_{i}} M_{l}$. From the arguments developed in the proof of Point (1), it is easy to see that $\left\{\left(T_{M_{i}} M_{l}, T \epsilon_{l i}\right)\right\}_{l \geq i}$ is a direct sequence of Banach bundles. Since we have $P_{l j i}=T \epsilon_{j i} \circ P_{j}$, we get a morphism $\bar{P}_{j i}=\lim _{l \geq j} P_{l j i}$ from $T_{M_{i}}^{\prime} M_{j}$ to $\lim _{l \geq j} T_{M_{i}} M_{j}=\bigcup_{l \geq j} T_{M_{i}} M_{l}$. Note that since we have $T M_{i} \subset T_{M_{i}} M_{i+1} \subset$ $\cdots \vec{\subset} T_{M_{i}} M_{j}$, then we also have

$$
\lim _{l \geq j} T_{M_{i}} M_{j}=\bigcup_{l \geq i} T_{M_{i}} M_{l}=T M_{\mid M_{i}}=T_{M_{i}} M .
$$

On one hand, recall that $\left\{\left(T_{M_{i}}^{\prime} M_{j}, T^{*} \epsilon_{j i} \mid T_{M_{i}}^{\prime} M_{j}\right)\right\}_{j>i}$ is a strong projective sequence of bundles over $M_{i}$ (see the second part of the proof of Point (2)). On the other hand, we have a family of morphisms $\bar{P}_{j i}: T_{M_{i}}^{\prime} M_{j} \rightarrow T_{M_{i}} M$ over $M_{i}$ such that $\bar{P}_{h i}=\bar{P}_{j i} \circ T^{*} \epsilon_{h j}$. 
This implies that we get a morphism

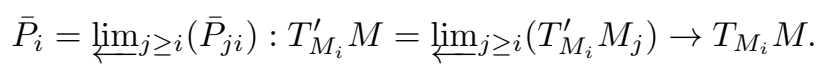

By construction, if $\left(x, \xi=\lim _{k \geq i}\left(\xi_{k}\right)\right) \in T_{M_{i}}^{\prime} M$ then

$$
\bar{P}_{i}(x, \xi)=\left(x, \lim _{k \geq i}\left(P_{k}\left(x, \xi_{k}\right)\right) .\right.
$$

Now recall that each $P_{k}$ is antisymmetric, i.e. in each fiber over $x$ we have the relation

$$
<\eta_{k}, P_{k}\left(x, \xi_{k}\right)>=-<\xi_{k}, P_{k}\left(x, \eta_{k}\right)>.
$$

Thus given two covectors $\left.\xi=\lim _{k \geq i}\left(\xi_{k}\right)\right)$ and $\left.\eta=\lim _{k \geq i}\left(\eta_{k}\right)\right)$ in $T_{x}^{\prime} M$, we obtain

$$
<\eta, \bar{P}_{i}(x, \xi)>=-<\xi, \bar{P}_{i}(x, \eta)>\text {. }
$$

Finally, since we have ascending sequences $\left\{T_{M_{i}}^{\prime} M\right\}_{i \in \mathbb{N}}$ and $\left\{T_{M_{i}} M\right\}_{i \in \mathbb{N}}$, from the construction of the sequence $\left\{\bar{P}_{i}\right\}_{i \in \mathbb{N}}$ of morphisms, we have $\bar{P}_{j}(x, \xi)=$ $\bar{P}_{i}(x, \xi)$ for all $j \geq i$; we then obtain a morphism $P: T^{\prime} M \rightarrow T M$ which is antisymmetric relatively to the canonical duality pairing between $T^{*} M$ and $T M$ in restriction to $T^{\prime} M \times T M$.

We now give a sketch of the proof of Theorem 3.3.4 in the case of direct limits.

According to Proposition 3.4.1, we have a vector subbundle $T^{\prime} M \rightarrow M$ of $T^{*} M \rightarrow M$ and a skew-symmetric morphism $P: T^{\prime} M \rightarrow T M$ and so is a partial Poisson anchor which defines an almost Poisson bracket $\{., .\}_{P}$.

Now, if $f=\lim _{\longrightarrow}\left(f_{i}\right)$ then $f_{i}=f \circ \bar{\epsilon}_{i}$ and $g_{i}=g \circ \bar{\epsilon}_{i}$ and so $d f_{i}=T^{*} \bar{\epsilon}_{i}(d f)$. Thus we have:

$$
\{f, g\}_{P} \circ \bar{\epsilon}_{i}=d f_{i}\left(T \bar{\epsilon}_{i}(P(d g))=d f_{i}\left(P_{i}\left(d g_{i}\right)=\left\{f_{i}, g_{i}\right\}_{P_{i}}=\left\{f \circ \bar{\epsilon}_{i}, g \circ \bar{\epsilon}_{i}\right\}_{P_{i}} .\right.\right.
$$

Since $\bar{\epsilon}_{i}=\bar{\epsilon}_{j} \circ \epsilon_{j i}$ according to Remark 3.3 .3 it follows that

$$
\{f, g\}_{P}=\lim _{\longrightarrow}\left\{f_{i}, g_{i}\right\}_{P_{i}} .
$$

Now, as each Poisson bracket $\{,\}_{P_{i}}$ satisfies the Jacobi identity, the same is true for $\{., .\}_{P}$ on $\mathcal{A}_{P}(M)=\lim \mathcal{A}\left(M_{i}\right)$. It follows that $\left(M, \mathcal{A}_{P}(M),\{,\}_{P}\right)$ is a partial Poisson manifold. Finally the Equation (3.11) means the $\bar{\epsilon}_{i}$ is a Poisson map. This ends the proof of Theorem 3.3.4 in the case of direct limits.

\subsection{Proof of Theorem 3.3.4 in the case of Inverse limit}

Let $\left\{\left(M_{i}, \mathcal{A}\left(M_{i}\right),\{,\}_{P_{i}}\right)\right\}_{i \in \mathbb{N}}$ be an inverse sequence of partial Poisson Banach manifolds. For $j>i$, we set $\tau_{j i}=\tau_{i} \circ \cdots \circ \tau_{j-1}: M_{j} \rightarrow M_{i}$. From the assumption in Definition 3.3.1 (2) the projective limit $M=\varliminf_{\varliminf}\left(M_{i}\right)$, is a Fréchet manifold; 
In particular $M$ is a convenient manifold. Note that since $\left\{\left(M_{i}, \tau_{i}\right)\right\}_{i \in \mathbb{N}}$ is an inverse sequence of Banach manifolds, this implies that, for all $i \in \mathbb{N}$, we have a surjective linear continuous map $\hat{\tau}_{i}: \mathbb{M}_{i+1} \rightarrow \mathbb{M}_{i}$ (cf. Remark 3.3.2). If we set $\hat{\tau}_{j i}=\hat{\tau}_{i} \circ \cdots \circ \hat{\tau}_{j-1}: \mathbb{M}_{j} \rightarrow \mathbb{M}_{i}$ then $\left\{\left(\mathbb{M}_{i}, \hat{\tau}_{j i}\right)\right\}_{j>i}$ is an inverse sequence of Banach spaces and $M$ is modelled on the Fréchet space $\mathbb{M}=\lim \left(\mathbb{M}_{i}\right)$. As in Gal], the set $\left\{\left(T M_{i}, T \tau_{j i}\right)\right\}_{j \geq i}$ is a strong projective sequence of Banach manifolds and $T M=\lim \left(T M_{i}\right)$ is the kinematic tangent bundle of the Fréchet manifold $M$ modelled on $\mathbb{M}$. Now, since $M$ is a Fréchet manifold, the dual convenient kinematic bundle $p^{*}: T^{*} M \rightarrow M$ is well defined and its typical fibre is the strong dual $\mathbb{M}^{*}$ of $\mathbb{M}$ (cf. KriMic, 33.1).

We identify $M$ with the set

$$
\left\{x=\left(x_{i}\right) \in \prod_{i \in \mathbb{N}^{*}} M_{i}: x_{i}=\tau_{j i}\left(x_{j}\right) \text { for } j>i \geq 1\right\}
$$

Since for each $j>i, \tau_{j i}: M_{j} \rightarrow M_{i}$ is a submersion, the transpose map $T^{*} \tau_{j i}: T_{\tau_{j i}(x)}^{*} M_{i} \rightarrow T_{x}^{*} M_{j}$ is a continuous linear injective map whose range is closed, for all $x \in M_{j}$. Now we have a submersion $\bar{\tau}_{i}: M \rightarrow M_{i}$ defined by $\bar{\tau}_{i}(x)=x_{i} \in M_{i}$ for each $x \in M$, and again the transpose map $T^{*} \bar{\tau}_{i}: T_{\bar{\tau}_{i}(x)}^{*} M_{i} \rightarrow$ $T_{x}^{*} M$ is a linear continuous injection whose range is closed. Therefore we have an ascending sequence $\left\{T_{\bar{\tau}_{i}(x)}^{*} M_{i}\right\}_{i \in \mathbb{N}}$ of closed Banach spaces. Since $T_{x} M$ is the projective limit of $\left.\left\{T_{\bar{\tau}_{i}(x)} M_{i}\right)\right\}$, each vector space $\underline{\lim }\left(T_{\bar{\tau}_{i}(x)}^{*} M_{i}\right)$ is the strong dual of $T_{x} M$ and is a convenient space (cf. [CabPel2]). In particular, we have $T_{x}^{*} M=\underline{\lim }\left(T_{\bar{\tau}_{i}(x)}^{*} M_{i}\right)$

Now from Definition 3.3 .1 Point (2), property (i), we have $T^{*} \tau_{j i}\left(T_{\bar{\tau}_{j i}(x)}^{*} M_{i}\right) \subset$ $T_{x}^{*} M_{j}$ for all $x \in M_{j}$. Therefore, with our previous identifications, $\left\{T_{\bar{\tau}_{i}(x)}^{\prime} M_{i}\right\}_{i \in \mathbb{N}}$ is an ascending sequence of closed Banach spaces contained in $T_{x}^{*} M$. It follows that $T_{x}^{\prime} M=\lim _{\longrightarrow}\left(T_{\bar{\tau}_{i}(x)}^{\prime} M_{i}\right)$ is a convenient subspace of $T_{x}^{*} M$. We set $T^{\prime} M=\bigcup_{x \in M} T_{x}^{\prime} M$ and $p^{\prime}: T^{\prime} M \rightarrow M$ the map defined by $p^{\prime}(x, \xi)=x$.

We then have:

\section{Proposition 3.5.1.}

1. $p^{\prime}: T^{\prime} M \rightarrow M$ is a convenient subbundle of the cotangent bundle $p^{*}$ : $T^{*} M \rightarrow M$.

2. For each $i \in \mathbb{N}$, there exists a canonical bundle morphism $\bar{T}^{\prime} \bar{\tau}_{i}: T^{\prime} M \rightarrow$ $T^{\prime} M_{i}$ over $\bar{\tau}_{i}$ such that $\bar{T}^{\prime} \bar{\tau}_{i}(x, \xi)=\left(x_{i}, \xi_{i}\right)$ if $x=\lim _{\longleftarrow}\left(x_{i}\right)$ and $\xi=\underset{\lim }{\longrightarrow}\left(\xi_{i}\right)$ where

$$
P_{i} \circ \bar{T}^{\prime} \bar{\tau}_{i}(x, \xi)=T \tau_{j i} \circ P_{j} \circ \bar{T}^{\prime} \bar{\tau}_{j}(x, \xi)
$$

for all $(x, \xi) \in T^{\prime} M$ and $j>i$. Then $P=\lim _{(}\left(P_{i} \circ \bar{T}^{\prime} \bar{\tau}_{i}\right)$ is a bundle morphism from $T^{\prime} M$ to $T M$ which is skew-symmetric (relatively to the canonical dual pairing between $T^{*} M$ and $T M$ in restriction to $T^{\prime} M \times T M$ ).

Proof of Proposition 3.5.1. . In this proof, we will consider an inverse sequence $\left\{\left(U_{i}, \phi_{i}\right)\right\}_{i \in \mathbb{N}}$ of charts such that $\left(U=\lim _{\longleftarrow}\left(U_{i}\right), \phi=\lim _{\longleftarrow}\left(\phi_{i}\right)\right)$ is a chart of $M$. 
Proof of Point (1)

At first we will show that $T_{M}^{*} M_{l}=\bigcup_{x \in M} T^{*} \bar{\tau}_{l}\left(T_{\bar{\tau}_{l}(x)}^{*} M_{i}\right)$ is the total space of a convenient bundle over $M$ which is nothing more than $p^{*}: T^{*} M \rightarrow M$ and that $T_{M}^{\prime} M_{l}=\bigcup_{x \in M} T^{*} \bar{\tau}_{l}\left(T_{\bar{\tau}_{l}(x)}^{\prime} M_{i}\right)$ is the total space of a convenient bundle $p^{\prime}: T^{\prime} M \rightarrow M$ which is also a closed subbundle of $p^{*}: T^{*} M \rightarrow M$.

Fix some chart $\left(U=\lim _{\longleftarrow}\left(U_{l}\right), \phi=\lim _{\longleftarrow}\left(\phi_{l}\right)\right)$ as previously, and for each $l \in \mathbb{N}$, consider

$$
\bigcup_{x \in U} T^{*} \bar{\tau}_{l}\left(T_{\bar{\tau}_{l}(x)}^{*} M_{l}\right)=T_{M}^{*} M_{l \mid U} \subset T^{*} M_{\mid U}
$$

Recall that we have $\bar{\tau}_{l}(U)=U_{l}$ and $\hat{\bar{\tau}}_{l} \circ \phi=\phi_{l} \circ \bar{\tau}_{l}$ where $\hat{\bar{\tau}}_{l}$ is the natural linear projection $\mathbb{M}=\lim \left(\mathbb{M}_{l}\right)$ on $\mathbb{M}_{l}$.

Therefore, if $\hat{\bar{\tau}}_{l}^{*}$ is the adjoint of $\hat{\bar{\tau}}_{l}$ then $\hat{\bar{\tau}}_{l}^{*}$ is injective and so $\mathbb{M}^{*}$ can be identified with the direct limit of the ascending sequence $\left\{\left(\mathbb{M}_{l}^{*}, \hat{\bar{\tau}}_{l}^{*}\right)\right\}_{l \in \mathbb{N}}$ of Banach spaces. It follows that $T^{*} \phi: \phi(U) \times \mathbb{M}^{*} \rightarrow T^{*} M_{\mid U}$ is a bundle isomorphism which is the inverse of the trivialization $T^{*} \phi^{-1}$ of the cotangent bundle over $U$. Moreover, on $T M_{\mid U}$, we also have

$$
T \hat{\bar{\tau}}_{l} \circ T \phi=T \phi_{l} \circ T \bar{\tau}_{l} .
$$

We then obtain

$$
T^{*} \phi \circ T^{*} \hat{\bar{\tau}}_{l}=T^{*} \bar{\tau}_{l} \circ T^{*} \phi_{l} \text { on }\left\{\hat{\bar{\tau}}_{l}(\phi(x))\right\} \times \mathbb{M}_{l}^{*} \text { for all } x \in U
$$

In particular

$$
T^{*} \phi \circ T^{*} \hat{\bar{\tau}}_{l}:\left\{\bar{\tau}_{l}(\phi(x))\right\} \times \mathbb{M}_{l}^{*} \rightarrow T^{*} \bar{\tau}_{l}\left(T_{\bar{\tau}_{l}(x)}^{*} M_{l}\right) \subset T_{x}^{*} M
$$

is a linear map for all $x \in U$.

But $T^{*} \hat{\bar{\tau}}_{l} \equiv \hat{\bar{\tau}}_{l}^{*}:\left\{\hat{\bar{\tau}}_{l}(t)\right\} \times \mathbb{M}_{l}^{*} \rightarrow\{t\} \times \mathbb{M}^{*}$ is an injective closed linear map for all $t \in \phi(U)$. Therefore, on the one hand, if we denote $\mathbb{E}_{l}=\hat{\bar{\tau}}_{l}^{*}\left(\mathbb{M}_{l}^{*}\right)$, then $\hat{\bar{\tau}}_{l}^{*}$ is an isomorphism from $\mathbb{M}_{l}^{*}$ onto the closed Banach subspace $\mathbb{E}_{l}$ of $\mathbb{M}^{*}$; so we can assume that $\mathbb{M}_{l}^{*}$ is contained in $\mathbb{M}^{*}$. With these identifications, the map $T^{*} \phi \circ T^{*} \hat{\bar{\tau}}_{l}$ is nothing but the natural inclusion of $\phi(U) \times \mathbb{M}_{l}^{*}$ into $\phi(U) \times \mathbb{M}^{*}$.

In this way, we obtain $T^{*} \phi\left(\phi(U) \times \mathbb{M}_{l}^{*}\right)=T_{M}^{*} M_{l \mid U} \subset T^{*} M_{\mid U}$. This implies that $T_{M}^{*} M_{l}$ is the total space of a closed trivial subbundle of $T^{*} M \rightarrow M$ with typical fiber $\mathbb{M}_{l}^{*}$.

Now, recall that $\mathbb{F}_{l}$ is a Banach subspace of $\mathbb{M}_{l}^{*}$. From Equation (3.12) and the assumptions of Definition 3.5.1, Point (2) and the previous arguments, we have

$$
T^{*} \phi\left(\phi(U) \times \mathbb{F}_{l}\right)=\bigcup_{x \in U} T^{*} \bar{\tau}_{l}\left(T_{\bar{\tau}_{l}(x)}^{\prime} M_{l}\right)=T_{M}^{\prime} M_{l_{\mid U}} \subset T^{*} M_{\mid U}
$$

Therefore $T_{M}^{\prime} M_{l}$ is also the total space of a closed trivial subbundle of $T^{*} M \rightarrow$ $M$ with typical fiber $\mathbb{F}$. So the proof of our affirmation is complete.

Since $\hat{\tau}_{j i}^{*}$ is a linear continuous closed inclusion of $\mathbb{M}_{i}^{*}$ into $\mathbb{M}_{j}^{*}$, via any chart $\left(U=\lim _{\longleftarrow}\left(U_{l}\right), \phi=\lim _{\longleftarrow}\left(\phi_{l}\right)\right)$ with the properties required at the beginning of the 
proof of Proposition 3.5.1 we can build an injective morphism $T_{j i}^{U}: T_{M}^{*} M_{i \mid U} \rightarrow$ $T_{M}^{*} M_{j \mid U}$ given by

$$
T_{j i}^{U}(x, \xi)=T^{*} \phi \circ \hat{\tau}_{j i}^{*} \circ T^{*} \phi^{-1}(x, \xi) \text { for all }(x, \xi) \in T_{M}^{*} M_{i \mid U}
$$

Moreover for any other chart $\left(U^{\prime}=\lim _{\longleftarrow}\left(U_{i}^{\prime}\right), \phi^{\prime}=\lim _{\longleftarrow}\left(\phi_{l}^{\prime}\right)\right)$ of this type with $U \cap U^{\prime} \neq \emptyset$, for all $(x, \xi) \in T_{M}^{*} M_{i U \cap U^{\prime}}$, we have

$$
T_{j i}^{U^{\prime}}(x, \xi)=T^{*} \phi^{\prime} \circ T^{*} \phi^{-1} \circ T_{j i}^{U} \circ T^{*} \phi \circ T^{*} \phi^{\prime-1}(x, \xi)
$$

We get an injective bundle morphism $T_{j i}: T_{M}^{*} M_{i} \rightarrow T_{M}^{*} M_{j}$ which is nothing but the inclusion of $T_{M}^{*} M_{i}$ into $T_{M}^{*} M_{j}$. In other words, $T_{M}^{*} M_{i} \rightarrow M$ is a Banach subbundle of $T_{M}^{*} M_{j}$ for $j \geq i$. Finally, from this construction, it follows that $\left\{\left(T_{M}^{*} M_{i}, T_{j i}\right)\right\}_{j \geq i}$ is an ascending sequence of Fréchet manifolds which has the direct limit chart property at every point of $\lim \left(T_{M}^{*} M_{i}\right)$. It follows that $\lim _{(}\left(T_{M}^{*} M_{i}\right)$ is a convenient manifold modelled on $\overrightarrow{\mathbb{M}} \times \mathbb{M}^{*}$; In particular we have $T^{*} M=\underline{\lim }\left(T_{M}^{*} M_{i}\right)$. Moreover, around each point $\left(x, \xi_{i}\right)$ in $T_{M}^{*} M_{i}$, there exists a chart $\left(T_{M}^{*} M_{i \mid U}, T^{*} \phi_{\mid\left\{T_{M}^{*} M_{i \mid U}\right\}}\right)$ where $(U, \phi)$ is a chart around $x$ which has the properties required at the begining of the proof of Proposition 3.5.1.

Therefore $\left(T^{*} M_{\mid U}=\lim _{\longrightarrow}\left(T_{M}^{*} M_{i \mid U}\right),(T \phi)^{*}=T^{*} \phi^{-1}=\lim _{\longrightarrow}\left(T^{*} \phi_{\mid\left\{T_{M}^{*} M_{i \mid U}\right\}}\right)\right)$ is a chart around $\left(x, \xi=\underline{\lim }\left(\xi_{i}\right)\right)$ in $T^{*} M$. This implies that $T^{*} M=\underline{\lim }\left(T_{M}^{*} M_{i}\right)$ is a convenient vector bundle over $M$ whose typical fiber is $\mathbb{M}^{*}=\underset{\lim }{\longrightarrow}\left(\mathbb{M}_{i}^{*}\right)$.

Clearly the same arguments can be applied to $\left\{\left(T_{M}^{\prime} M_{i}, T_{j i} \mid T_{M}^{\prime} M_{i}\right)\right\}_{j \geq i}$ and so we have $T^{\prime} M=\underline{\lim }\left(T_{M}^{\prime} M_{i}\right)$ and we get a convenient vector bundle $p^{\prime}: T^{\prime} M \rightarrow M$ with typical fiber $\mathbb{F}=\underline{\lim }\left(\mathbb{F}_{i}\right)$.

Proof of Point (2)

For each $i \in \mathbb{N}$, consider the bundle $\bar{p}_{i}^{\prime}: T_{M}^{\prime}{ }^{*} M_{i} \rightarrow M$. Obviously, this bundle is nothing but the pull back over $\bar{\tau}_{i}: M \rightarrow M_{i}$ of the bundle $p_{i}^{\prime}: T^{\prime} M_{i} \rightarrow M_{i}$. Therefore we have a bundle morphism $T^{\prime} \bar{\tau}_{i}$ over $\bar{\tau}_{i}$ from $T_{M}^{\prime} M_{i}$ to $T^{\prime} M_{i}$ such that its restriction to any fiber is an isomorphism whose inverse is $T^{*} \bar{\tau}_{i}$ in restriction to $T_{\bar{\tau}_{i}(x)}^{\prime} M_{i}$. Since $T^{*} M=\underline{\lim }\left(T_{M}^{*} M_{i}\right)$, we have an injective bundle morphism $T_{i}: T_{M}^{*} M_{i} \rightarrow T^{*} M$ which is the natural inclusion. Note that for all $j>i$ we have $T_{j}=T_{j i} \circ T_{i}$, where $T_{j i}: T_{M}^{\prime} M_{i} \rightarrow T_{M}^{\prime} M_{j}$ is the natural inclusion (cf. Proof of Pont(1)).

Now from the relation $\bar{\tau}_{i}=\bar{\tau}_{j} \circ \tau_{j i}$, for $j \geq i$, we obtain for all $\left(x, \xi_{i}\right) \in T_{M}^{\prime} M_{i}$ :

$$
T^{\prime} \bar{\tau}_{j} \circ T_{j i}\left(x, \xi_{i}\right)=T^{\prime} \bar{\tau}_{i} \circ T^{*} \tau_{j i}\left(x, \xi_{i}\right)
$$

Now, for $(x, \xi) \in T^{\prime} M$, there exists an integer $i \in \mathbb{N}$ such that $(x, \xi)$ belongs to $T_{M}^{\prime} M_{i}$; so $(x, \xi)$ also belongs to $T_{M}^{\prime} M_{j}$ for $j>i$ and we obtain

$$
T^{\prime} \bar{\tau}_{j}(x, \xi)=T^{\prime} \bar{\tau}_{i} \circ T^{*} \tau_{j i}(x, \xi) .
$$


Finally, from the assumption (ii) of Point (2) in Definition 3.3.1, by induction on $j>i$, we get the following commutative diagram:

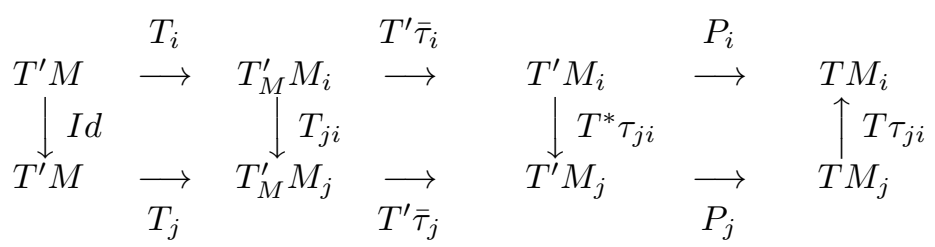

We set $\bar{P}_{i}(x, \xi)=P_{i}\left(\bar{\tau}_{i}(x), \bar{T}^{\prime} \bar{\tau}_{i} \circ T_{i}(\xi)\right)$. Note that $\bar{P}_{i}$ is a bundle morphism from $T^{\prime} M$ into $T M_{i}$ over $\bar{\tau}_{i}$. According to the previous commutative diagram, we obtain

$$
\bar{P}_{j}(x, \xi)=T \tau_{j i} \circ \bar{P}_{i}(x, \xi)
$$

Since $T M=\lim \left(T M_{i}\right)$ we get bundle morphism $P=\lim _{\longleftarrow}\left(\bar{P}_{i}\right): T^{\prime} M \rightarrow T M$. In particular we have $T \bar{\tau}_{i} \circ P=\bar{P}_{i}$. Now we can remark that $\bar{T}^{\prime} \bar{\tau}_{i}=T_{i} \circ T^{\prime} \bar{\tau}_{i}$ is a bundle morphism over $\bar{\tau}_{i}$ such that $\bar{T}^{\prime} \bar{\tau}_{i}(x, \xi)=\left(x_{i}, \xi_{i}\right)$ if $x=\varliminf_{\varliminf}\left(x_{i}\right)$ and $\xi=\varliminf_{\longrightarrow}\left(\xi_{i}\right)$ and also $\bar{P}_{i}=P_{i} \circ \bar{T}^{\prime} \bar{\tau}_{i}$.

It remains to prove that $P$ is skew-symmetric. Since $P_{i}$ is skew-symmetric relatively the canonical pairing $<,>_{i}$ between $T^{*} M_{i}$ and $T M_{i}$ we have

$$
<\bar{T}^{\prime} \bar{\tau}_{i}(x, \eta), \bar{P}_{i}(x, \xi)>_{i}=-<\bar{T}^{\prime} \bar{\tau}_{i}(x, \xi), \bar{P}_{i}(x, \eta)>_{i}
$$

If $<,>$ denotes the canonical pairing between $T^{*} M$ and $T M$, for any kinematic differential form $\eta=\underline{\lim }\left(\eta_{i}\right)$ and $\xi=\underline{\lim }\left(\xi_{i}\right)$ on $M$ which are sections of $p^{\prime}: T^{\prime} M \rightarrow M$. Note in fact, $\eta=\bar{\tau}_{i}^{*} \eta_{i}$ and $\vec{\xi}=\bar{\tau}_{i}^{*} \xi_{i}$, for any $i \in \mathbb{N}$. There exists an integer $i$ such that $\eta$ and $\xi$ are sections of $T_{M}^{\prime} M_{i}$. Therefore

$<\eta, P(\xi)>=<\bar{\tau}_{i}^{*} \eta_{i}(P(x, \xi))>=\eta_{i}\left(T \bar{\tau}_{i} \circ P(x, \xi)\right)=\eta_{i}\left(\bar{P}_{i}(x, \xi)\right)=<\bar{T}^{\prime} \bar{\tau}_{i}(x, \eta), \bar{P}_{i}(x, \xi)>_{i}$

The Equation (3.13) implies that $P$ is skew-symmetric.

We now give the sketch of the Proof of Theorem 3.3.4 in the case of inverse limits.

According to Proposition 3.5.1 to the skew-symmetric morphism $P: T^{\prime} M \rightarrow$ $T M$ is associated the algebra $\mathcal{A}(M)$ of smooth functions $f: M \rightarrow \mathbb{R}$ whose differential $d f$ is a section of $T^{\prime} M \rightarrow M$. Denote by $\{,\}_{P}$ the associated almost bracket. Now, if $f=\lim \left(f_{i}\right)$ and $g=\lim \left(g_{i}\right)$ then $f=f_{i} \circ \bar{\tau}_{i}$ and $g=g_{i} \circ \bar{\tau}_{i}$ and so $d f=T^{*} \bar{\tau}_{i}\left(d f_{i}\right)$ and $\overline{d g}=T^{*} \bar{\tau}_{i}\left(d g_{i}\right)$. Using the relation of compatibility

$$
P_{i}=T \tau_{j i} \circ P_{j} \circ T^{*} \tau_{j i}
$$

and the relations $d f_{j}=T^{*} \tau_{j i}\left(d f_{i}\right), d g_{j}=T^{*} \tau_{j i}\left(d g_{i}\right),\left\{f_{i}, g_{i}\right\}_{P_{i}}=d f_{i}\left(P_{i}\left(d g_{i}\right)\right)$, we obtain (cf. Remark 3.3.3)

$$
\left\{f_{j}, g_{j}\right\}_{P_{j}}=\left\{f_{i}, g_{i}\right\}_{P_{i}} \circ \tau_{j i}
$$


We also have:

$$
\left\{f_{i} \circ \bar{\tau}_{i}, g_{i} \circ \bar{\tau}_{i}\right\}_{P}=\left\{f_{i}, g_{i}\right\}_{P_{i}} \circ \bar{\tau}_{i} .
$$

Thus $\{f, g\}_{P}=\lim _{\longleftarrow}\left\{f_{i}, g_{i}\right\}_{P_{i}}$ is defined on the subalgebra $\mathcal{A}_{P}(M)=\lim \mathcal{A}\left(M_{i}\right)$ of $\mathcal{A}(M)$.

Now, as each Poisson bracket $\{,\}_{P_{i}}$ satisfies the Jacobi identity, the same is true for $\{,\}_{P}$ on $\mathcal{A}_{P}(M)$ and so $\left(M, \mathcal{A}_{P}(M),\{,\}_{P}\right)$ is a partial Poisson structure. Finally, Equation (3.14) means that $\bar{\tau}_{i}$ is a Poisson map. This ends the proof of Theorem 3.3.4 in the case of inverse limits.

\section{Existence of almost symplectic foliation for di- rect limit partial Poisson Banach manifolds}

Before proving a result of the same type as Theorem 2.4.10 for a direct sequence of partial Poisson Banach manifolds, we need preliminaries on partial Banach Poisson manifolds.

Let $\pi: E \rightarrow M$ be a Banach bundle. Classically, a Koszul connection on $E$ is a $\mathbb{R}$-bilinear map $\nabla: \Gamma(T M) \times \Gamma(E) \rightarrow \Gamma(E)$ which, for any function $\phi$ on $M, X \in \Gamma(M)$ and $\sigma \in \Gamma(E)$, fulfils the following properties:

$$
\begin{gathered}
\nabla_{X}(\phi \sigma)=d \phi(X) \sigma+\phi \nabla_{X} \sigma \\
\nabla_{\phi X} \sigma=\phi \nabla_{X} \sigma .
\end{gathered}
$$

Unfortunately, in general, a Koszul connection may be not localizable in the following sense:

Since any local section of $E$ (resp. any local vector field on $M$ ) cannot be always extended to a global section of $E$ (resp. to a global vector field on $M)$, the previous operator $\nabla$ cannot always induce a (local) operator $\nabla^{U}$ : $\Gamma\left(T M_{\mid u}\right) \times \Gamma\left(E_{\mid U}\right) \rightarrow \Gamma\left(E_{\mid U}\right)$. Therefore, in this work, a Koszul connection will always assumed to be localizable in this sense (For more details see CabPel2] section 5.2).

Now consider a direct sequence of partial Poisson Banach manifolds we have:

Theorem 4.0.1. Let $\left\{\left(M_{i}, \mathcal{A}\left(M_{i}\right),\{,\}_{P_{i}}\right)\right\}_{i \in \mathbb{N}}$ be a direct sequence of partial Poisson Banach manifolds. Assume that, for each $i \in \mathbb{N}$, the following assumptions are satisfied:

(1) There exists a Koszul connection on each $T^{\prime} M_{i}$;

(2) Over each point $x \in M_{i}$ the kernel of $P_{i}$ is supplemented in the fiber $p_{i}^{\prime-1}(x)$ and the distribution $P\left(T^{\prime} M_{i}\right)$ is closed;

(3) There exists $j_{i} \geq i$ such that, for any $x \in M$, we have $P_{j}\left(T_{x}^{\prime} M_{j}\right)=$ $P_{j_{i}}\left(T_{x}^{\prime} M_{j_{i}}\right)$ for $j \geq j_{i}$. 
Then we have:

1. Each distribution $P_{i}\left(T^{\prime} M_{i}\right)$ on $M_{i}$ is integrable and the direct limit $\Delta=$ $\underset{\lim }{\longrightarrow} P_{i}\left(T^{\prime} M_{i}\right)$ is also an integrable distribution on $M=\underset{\lim }{\longrightarrow}\left(M_{i}\right)$.

2. For any $x=\lim \left(x_{i}\right)$, the maximal leaf though $x$ is a weak convenient manifold of $M$ and there exists a leaf $N_{i}$ of $P_{i}\left(T^{\prime} M_{i}\right)$ in $M_{i}$ through $x_{i}$, such that the sequence $\left(N_{i}\right)_{i \in \mathbb{N}^{*}}$ is an ascending sequence of Banach manifolds whose direct limit $N=\underline{\lim }\left(N_{i}\right)$ is an integral manifold of $\Delta$ though $x$.

3. The natural almost symplectic structure $\left(N, \omega_{N}\right)$ on a leaf $N$ is such that

$$
\omega_{N}=\lim _{\longleftarrow}\left(\omega_{N_{i}}\right)
$$

Note that the condition (3) is always satisfied in the following cases:

(-) Each manifold $M_{i}$ is finite dimensional;

(-) Each bundle $T^{\prime} M_{i}$ has a finite dimensional fiber;

(-) Each morphism $P_{i}$ has finite rank.

Therefore we have:

Corollary 4.0.2. Let $\left\{\left(M_{i}, \mathcal{A}\left(M_{i}\right),\{,\}_{P_{i}}\right)\right\}_{i \in \mathbb{N}}$ be a direct sequence of Poisson finite dimensional manifolds. Then all conclusions of Theorem 4.0 .1 are valid.

Proof. We will use the notations and partial results of subsection 3.4

At first, from Point (1) of Definition 3.3.1, for any $j>i$, we have

$$
\Delta_{j i}=P_{j}\left(\left(T_{M_{i}}^{\prime} M_{j}\right) \subset P_{i}\left(T^{\prime} M_{i}\right)=\Delta_{i}\right.
$$

Let $\mathfrak{P}_{M_{j}}$ be the sheaf of local sections associated to the partial Poisson structure $\left(M_{j}, \mathcal{A}\left(M_{j}\right),\{,\}_{P_{j}}\right)$ as defined in Proposition 2.3.6. According to the proof of Theorem [2.3.6, $\mathfrak{P}_{M_{j}}$ is a generating set for sections of the anchored bundle $\left(T^{\prime} M_{j}, M_{j}, P_{j}\right)$ so the same property is true for the restriction $\mathfrak{P}_{M_{i}}^{j}$ of $\mathfrak{P}_{M_{j}}$ to $M_{i}$ for the anchored $\left(T_{M_{i}}^{\prime} M_{j}, M_{i}, P_{j}\right)$ since $M_{i} \subset M_{j}$ and according to (4.1). From the properties of sheaf of Lie brackets $[., .]_{P_{j}}$ the anchor $P_{j}$ gives rise to a Lie morphism on $\mathfrak{P}_{M_{j}}$ this property also true for its restriction to $\mathfrak{P}_{M_{i}}^{j}$, and the kernel of $P_{j}$ is supplemented in each fiber over each point of $M_{i} \subset M_{j}$ Therefore, by application of Corollary 2.4.8, the distribution $\Delta_{j i}$ is integrable on $M_{i}$.

For $i$ fixed, on $M_{i}$, we have a decreasing sequence of smooth distribution:2

$$
\Delta_{i}=\Delta_{i i} \supset \cdots \supset \Delta_{j i} \supset \cdots
$$

and we set $\bar{\Delta}_{i}=\cap_{j>i} \Delta_{j i}$. Note that since $\epsilon_{j i}: M_{i} \rightarrow M_{j}$ is the inclusion and $T_{M_{i}}^{\prime} M_{j} \subset T_{M_{i}}^{*} M_{j}$, we have $\epsilon_{j i}(x)=x$ and $T_{x}^{*} \epsilon_{j i}(\xi)=\xi_{\mid T_{x} M_{i}}$ for all $x \in M_{i}$ and

\footnotetext{
${ }^{2}$ Recall that a distribution $\Delta^{\prime}$ is contained in a distribution $\Delta$ on $M$ if, for any $x \in M$, $\Delta_{x}^{\prime} \subset \Delta_{x}$
} 
$\xi \in T_{x}^{\prime} M_{j}$. Therefore from Hahn-Banach theorem $T_{x}^{*} \epsilon_{j i}$ is surjective.

On the one hand, since $T_{x} M_{i} \subset T_{x} M_{i+1} \subset \cdots \subset T_{x} M_{j} \subset \cdots \subset T_{x} M$, we can choose a norm \|\|$_{j}$ on $T_{x} M_{j}$ for all $j \geq i$ such that \|\|$_{j+1} \leq\|\|_{j}$ for all $j \geq i$; In particular, the operator norm of $T_{x} \epsilon_{j i}$ is bounded by 1 . We then obtain a canonical norm \|\|$_{j}^{*}$ on $T_{x}^{*} M$ and so the operator norm of $T_{x}^{*} \epsilon_{j i}$ is bounded by 1 for all $j>i$. According to Property (ii) of Point (1) in Definition 3.3.1. all these considerations imply that the operator norm of $P_{j}$ is bounded by the operator norm of $P_{i}$ for $j \geq i$.

On the other hand, if we consider the Banach quotient space $T_{x}^{\prime} M_{j} / \operatorname{ker}\left(P_{j}\right)_{x}$, according to Proposition 2.4.5 each vector space $\left(\Delta_{j i}\right)_{x}$ has its own Banach space structure which is isomorphic to $T_{x}^{\prime} M_{j} / \operatorname{ker}\left(P_{j}\right)_{x}$ and so the space $\left(\bar{\Delta}_{i}\right)_{x}$ is provided with a Fréchet structure induced by the sequence of Banach spaces $\left(\Delta_{j i}\right)_{x}$. In fact, $\left(\bar{\Delta}_{i}\right)_{x}$ is a Banach space.

Fix some $x_{0} \in M_{i}$ and denote by $N_{j i}$ the maximal leaf of $\Delta_{j i}$ through $x_{0}$. We then have the following sequence of (weak) Banach submanifolds modelled on the previous Banach structure on $\left(\Delta_{j i}\right)_{x_{0}}$ and we have

$$
N_{i i} \supset \cdots \supset N_{j i} \supset \cdots
$$

Set $\bar{N}_{i}=\cap_{j \geq i} N_{j i}$. We will show that $\bar{N}_{i}$ is a Banach manifold modelled on the Banach space $\left(\bar{\Delta}_{i}\right)_{x}$.

Fix $x \in \bar{N}_{i} \subset N_{j i}$. Note that if $\left(\bar{\Delta}_{i}\right)_{x}=\{0\}$ then $\bar{N}_{i}=\{x\}$ and we have nothing to prove. From now on, we assume that $\operatorname{dim}\left(\Delta_{j i}\right)_{x}>0$.

Consider a chart $\left(U=\lim _{(}\left(U_{j}\right), \phi=\lim _{(}\left(\phi_{i}\right)\right)$ around $x$ in $M$ which satisfies Property (iii) of point (1) of Definition $\overrightarrow{3.3 .1}$ Recall that for each $j$, if $V_{j}=$ $\phi_{j}\left(U_{j}\right)$, we have a trivialization $T^{*} \phi_{j}^{-1}: V_{j} \times \mathbb{F}_{j} \rightarrow T_{U_{j}}^{\prime} M_{j}$. Since $U_{i} \subset U_{j}$ for $i \leq j$, we get a trivialization $\Theta_{j}: U_{i} \times \mathbb{F}_{j} \rightarrow T_{U_{i}}^{\prime} M_{j}$. Now, as $\widehat{\mathcal{M}}_{P_{j}}\left(M_{i}\right)$ is a generating set for the anchored bundle $\left(T_{M_{i}}^{\prime} M_{j}, M_{i}, P_{j}\right)$ and $\operatorname{ker}\left(P_{j}\right)_{x}$ is supplemented, if we have $T_{x}^{\prime} M_{j}=\operatorname{ker}\left(P_{i}\right)_{x} \oplus \mathbb{S}_{j}$ then the vector fields $X_{j}(\alpha)=$ $P_{j} \circ \Theta_{j}(, \alpha)$ belong to $\mathfrak{P}_{M_{i}}$ for all $\alpha \in T_{x}^{\prime} M_{j}$. Now according to Proposition 2.4.9. there exists a ball $B_{j}\left(0, r_{j}\right)$ in $\mathbb{S}_{j}$ such that

- the map $\Phi_{j}(\alpha)=\phi_{1}^{X_{j}(\alpha)}(x)$ is defined for $\alpha \in B_{j}\left(0, r_{j}\right)$;

- there exists $0<\delta_{j} \leq r_{j}$ such that $\Phi_{j}: B_{j}\left(0, \delta_{j}\right) \rightarrow M_{i}$ is a weak injective immersion;

- $\Phi_{j}\left(B_{j}\left(0, \delta_{j}\right)\right)$ is an integral manifold of $\Delta_{j i}$ through $x$.

Note that, in particular, $\Phi_{j}\left(B\left(0_{j}, \delta_{j}\right)\right)$ is an open set in $N_{j i}$. Now from the previous choice of $\left\{\left(U_{j}, \phi_{j}\right)\right\}_{j \in \mathbb{N}}$, on $U_{i}$, for all $\alpha \in \mathbb{S}_{j}$, we have:

$$
T^{*} \epsilon_{j i} \circ \Theta_{j}(, \alpha)=\Theta_{i}\left(, T_{x}^{*} \epsilon_{j i}(\alpha)\right)
$$

and so we get

$$
X_{j}(\alpha)=P_{j} \circ \Theta_{j}(, \alpha)=T \epsilon_{j i} \circ P_{i} \circ T^{*} \epsilon_{j i}\left(\Theta_{j}(, \alpha)\right)=T \epsilon_{j i} \circ X_{i}\left(T_{x}^{*} \epsilon_{j i}(\alpha)\right) .
$$


Therefore $X_{j}(\alpha)$ is tangent to $\Delta_{j i}$ and so we have:

$$
\forall \alpha \in B_{j}\left(0, r_{j}\right) \subset \mathbb{S}_{j}, \quad \Phi_{j}(\alpha)=\Phi_{i}\left(T_{x}^{*} \epsilon_{j i}(\alpha)\right)
$$

But we have $T_{x}^{*} \epsilon_{j i}\left(T_{x}^{\prime} M_{j}\right) \subset T_{x}^{\prime} M_{i}$ and $T_{0} \Phi_{j}$ is an isomorphism from $\mathbb{S}_{j}$ onto $\left(\Delta_{j i}\right)_{x}$. According to (4.2), it follows that $\left.T_{x}^{*} \epsilon_{j i}\right|_{\mathbb{S}_{j}}$ is a continuous injective linear map into $\mathbb{S}_{i}$. Again according to (4.2), we deduce that $r_{j} \geq r_{i}$ and $\delta_{j} \geq \delta_{i}$. For $j \geq i$, we set $\mathbb{S}_{j i}=T^{*} \epsilon_{j i}\left(\mathbb{S}_{j}\right) \subset \mathbb{S}_{i}, \bar{B}_{j i}\left(0, \delta_{i}\right)=T^{*} \epsilon_{j i}\left(B_{j}\left(0, \delta_{j}\right)\right) \cap$ $B_{i}\left(0, \delta_{i}\right), W_{j}=\Phi_{j}\left(B\left(0, \delta_{j}\right)\right.$ and $W_{j i}=W_{j} \cap W_{i}$. Then from the previous considerations, $\mathbb{S}_{j i}$ is isomorphic to $\left(\Delta_{j i}\right)_{x}, W_{j i}$ is an open set in $N_{j i}$ around $x$ and $\left(W_{j i},\left(\Phi_{i \mid B_{j i}\left(0, \delta_{i}\right)}\right)^{-1}\right)$ is a chart for $N_{j i}$ around $x$. We equip $\mathbb{S}_{j i}$ with the structure of Banach space such that $T_{x}^{*} \epsilon_{j i} \mathbb{S}_{j}$ is an isometry when we put on $\mathbb{S}_{j}$ the norm \|\|$_{j}^{*}$ induced from the norm \|\|$_{j}^{*}$ defined previously on $T_{x}^{*} M$. Then

$\overline{\mathbb{S}}_{i}=\cap_{j \geq i} \mathbb{S}_{j i}$ is then provided with a Banach structure. Then, from our previous construction, according to Gal, we obtain a Banach manifold structure on $\bar{N}_{i}$ modelled on $\overline{\mathbb{S}}_{i}$.

\section{References}

[Ana] M. Anastasiei, Banach Lie algebroids, arXiv:1003.1263 [math.DG].

[BGT] D. Beltiţă, T. Goliński, A-B. Tumpach, Queer Poisson brackets arxiv.org/abs/1710.03057v1.

[CabPel1] P. Cabau, F. Pelletier, Almost Lie structures on an anchored Banach bundle, Journal of Geometry and Physics 62 (2012) 2147-2169.

[CabPel2] P. Cabau, F. Pelletier, Integrability on Direct Limits of Banach Manifolds, arXiv:1408.3715 [math.DG], à paraître dans Annales de la Faculté des Sciences de Toulouse.

[DGV] C.T.J. Dodson, G. Galanis, E. Vassiliou, Geometry in a Fréchet Context: A projective Limit Approach, London Mathematical Society Lecture Note Series 428. Cambridge University Press, 2015.

[DuZu] J.-P. Dufour, N.T. Zung, Linearization of Nambu structures. Compositio Math. 117 (1999), no. 1, 77-98.

[EgeWur] M. Egeileh, T. Wurzbacher, Infinite-Dimensional Manifolds as Ring Spaces, Publications of the Research Institute for Mathematical Sciences, Volume 53, Issue 1 (2017) 187-209.

[FroKri] A. Frölicher, A. Kriegl, Linear Spaces and Differentiation Theory, Pure and Applied Mathematics, J. Wiley, Chichester 1988.

[Kol] K. Kolev, Poisson brackets in Hydrodynamics, Discrete and Continuous Dynamical Systems - Series A, American Institute of American Science (AIMS), 2007, 19 (3) 555-574. 
[Gal] G.-N. Galanis, Limits of Banach vector bundles Portugaliae Mathematica, vol 55, Fasc.1 (1998).

[KriMic] A. Kriegel, P.W. Michor, The convenient Setting of Global Analysis (AMS Mathematical Surveys and Monographs) 531997.

[Marl] C.-M. Marle, Lie group actions on a canonical manifold. In: Symplectic geometry. Res. Notes in Math., 80, Pitman, Boston, Mass.London, 1983, pp. 144-166.

[Mars] J.E. Marsden, Darboux's theorem fails for weak symplectic forms. Proc. Amer. Math. Soc. 32 (1972), 590-592.

[NeSaTh] K.-H. Neeb, H. Sahlmann, T. Thiemann, Weak Poisson structures on infinite dimensional manifolds and hamiltonian actions, arXiv:1402.6818v1 [math.DG].

[OdzRat] A. Odzijewicza, T. S. Ratiu, Induction for weak symplectic Banach manifolds, Journal of Geometry and Physics, 58 (2008) 701-719.

[Olv] P.J. Olver, Applications of Lie groups to differential equations. Second edition. Graduate Texts in Mathematics, 107. Springer-Verlag, New York, 1993.

[Pel] F. Pelletier, Integrability of weak distributions on Banach manifolds, Indagationes Mathematicae 23 (2012) 214-242.

[Rat] T. S. Ratiu, Coadjoint orbits and the beginnings of a geometric representation theory in Developments and trends in infinitedimensional Lie theory, Pianzola, Neeb Eds., 417-457, Progr. Math., 288, Birkhaüser Boston, Inc. Boston, MA, 2011.

[Tum] A. B. Tumpach, Banach Poisson Lie Groups and Bruhat Poisson Structure of the Restricted Grassmannian. Communications in Mathematical Physics 373(4) (2020) 795-858.

[Vai] I. Vaisman, Hamiltonian vector fields on almost symplectic manifolds, J. Math. Phys. 54, 092902 (2013).

[Wei] A. Weinstein, The local structure of Poisson manifolds, J. Diff. Geom (1983) 523-557. 